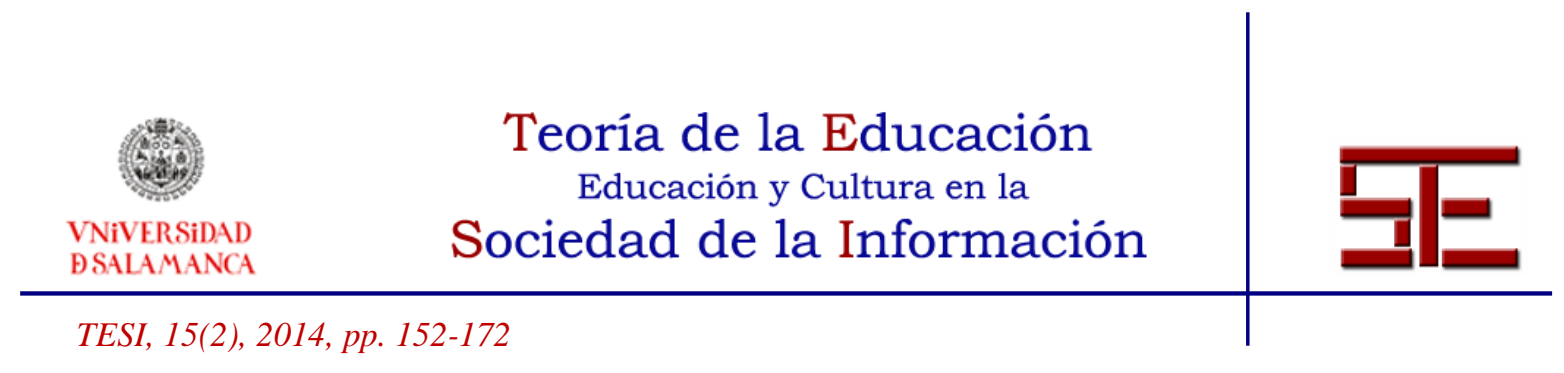

\title{
ANÁLISIS DE LA CONDUCTA VOCACIONAL EN ESTUDIANTES DE PSICOLOGÍA
}

Resumen: El Espacio Europeo de Educación Superior (EEES) viene apoyando la relevancia de situar la orientación vocacional en un lugar destacado en el marco de la educación universitaria. Para que esta orientación pueda ser planificada adecuadamente necesitamos conocer sus posibles contenidos evaluando los intereses vocacionales de los alumnos de cada centro. El objetivo del presente trabajo consistió en analizar los indicadores de la conducta vocacional de los estudiantes universitarios de Psicología, su evolución y su comparación con datos de otras poblaciones universitarias. Metodología. Los participantes fueron 329 alumnos de Psicología de la USAL (248 de segundo curso y 81 de quinto) que respondieron al Cuestionario de Biodatos Universitarios (Rocabert, 2005). En todos los casos se partió de un nivel de significación de $\alpha=0,05$ llevándose a cabo pruebas de comparación de muestras utilizando las técnicas U de Mann-Whitney y Análisis de Contingencia. Resultados. Se detectaron diferencias significativas entre los alumnos de segundo y de quinto, así como con la población universitaria general recogida por Rocabert, Descals y Gómez (2007). En general, los alumnos de Psicología inician sus estudios con un elevado interés y motivación, tomando decisiones académicas en función de la especialidad a la que se quieren dedicar. Sin embargo, en los últimos cursos detectamos una menor satisfacción por los estudios, más dificultades a la hora de decidir qué quieren hacer y mayor demanda de información para poder elegir la opción que más les conviene. Conclusiones. A pesar de la elevada motivación de los estudiantes de Psicología, a medida que su incorporación al mundo laboral es más cercana, requieren más orientación para tomar decisiones relacionadas con la especialización o el mundo profesional.

Palabras clave: Orientación universitaria; conducta vocacional; biodatos; motivación académica; toma de decisiones; practicum; enseñanza superior.

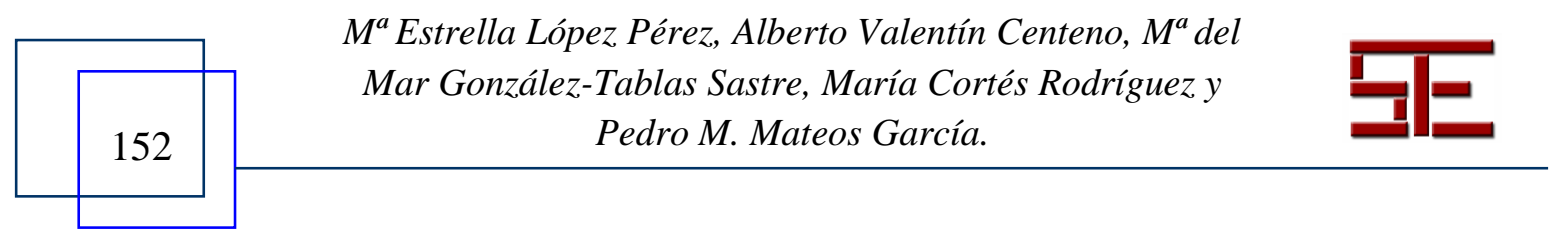




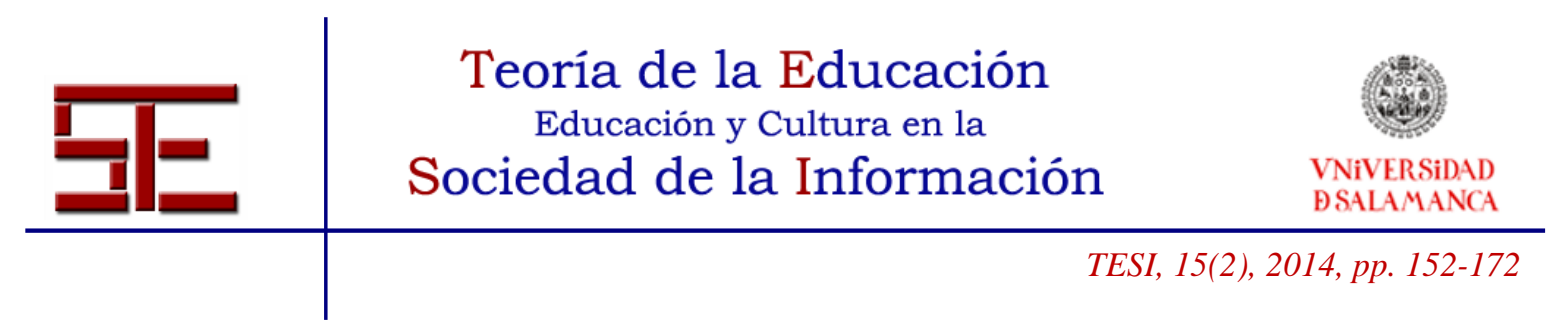

\title{
VOCATIONAL BEHAVIOR ANALYSIS IN PSYCHOLOGY STUDENTS
}

\begin{abstract}
Introduction. The European Higher Education Area (EHEA) is supporting gain relevance of vocational guidance into the frame of University Education. In order to a better planning of this guidance we need to know his contents evaluating student vocational interests of each center. The aim of the study is to analyze the indicators of Psychology students vocational behavior and his evolution and comparing those results with data of another students population. Methodology. The 329 psychology students participants from the University of Salamanca (248 in the second year and 81 in the fifth) answered the questionnaire of university biodata (Rocabert, 2005). In all cases we took a significance level of $\alpha=0.05$ carrying out samples comparison tests using $U$ de Mann-Whitney techniques and contingency analysis. Results: The present study found significant differences between second and fifth psychology grade students and with general university population data collected by Rocabert, Descals and Gómez (2007). In general, psychology students begin their degrees with a high level interest and motivated; they are making decisions based on the academic specialty they want to work in. However, for last year students group (fith year students) we detected a lower satisfaction in their studies, more difficulties in deciding what they want to do and a greater demand of information in order to choose the advisablest option for them. Conclusions. Despite the high motivation of psychology students, the nearer is his integration into the job market the higher is the need of guidance to help them to take decisions concerning specialization or the professional world.
\end{abstract}

Key words: University guidance; vocational behavior; biodata; academic motivation; decision making; practicum; higher education.

$M^{a}$ Estrella López Pérez, Alberto Valentín Centeno, $M^{a}$ del Mar

González-Tablas Sastre, María Cortés Rodríguez y Pedro M.

Mateos García. 


\title{
ANÁLISIS DE LA CONDUCTA VOCACIONAL EN ESTUDIANTES DE PSICOLOGÍA
}

Fecha de recepción: 18/01/2014; fecha de aceptación: 01/04/2014; fecha de publicación: 30/06/2014

Ma Estrella López Pérez

practpsi@usal.es

Universidad de Salamanca

\author{
Alberto Valentín Centeno \\ avc@usal.es \\ Universidad de Salamanca
}

Ma del Mar González-Tablas Sastre.

marola@usal.es

Universidad de Salamanca

María Cortés Rodríguez

mariacortes@usal.es

Universidad de Salamanca

Pedro M. Mateos García

pmateos@usal.es

Universidad de Salamanca

\section{1.- INTRODUCCIÓN}

El derecho a la orientación de los estudiantes ha sido matizado por el Real Decreto 1393/2007, de 29 de octubre, por el que se establece la Ordenación de las Enseñanzas

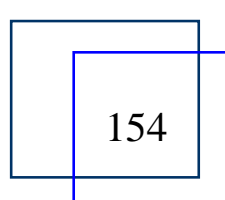

$M^{a}$ Estrella López Pérez, Alberto Valentín Centeno, $M^{a}$ del

Mar González-Tablas Sastre, María Cortés Rodríguez y

Pedro M. Mateos García.

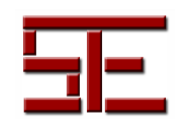




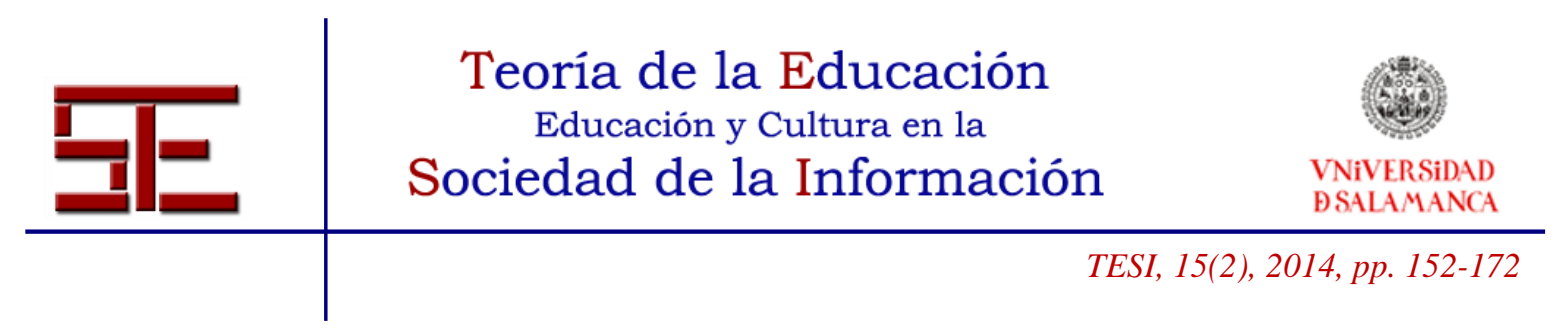

Universitarias Oficiales, en su artículo 14.2, convirtiendo el derecho de los estudiantes en una obligación para la Universidad, fijando en sus programas curriculares como imperativos "sistemas accesibles de información y procedimientos de acogida y orientación de los estudiantes de nuevo ingreso para facilitar su incorporación a las enseñanzas universitarias correspondientes".

Sin embargo, la realidad apunta que la Universidad ofrece una orientación más precaria y desigual que la que se brinda en los estudios previos, variando en función de los países y de las universidades (Salmerón, 2001) y centrándose, fundamentalmente, en el asesoramiento previo al ingreso en la Universidad.

En la última década y a raíz de los cambios requeridos por el Espacio Europeo de Educación Superior (EEES), son diversas las publicaciones que apoyan y proponen que la orientación necesita un lugar destacado en este contexto. Entre otros, el Informe Universidad 2000 (Bricall, 2000) especifica que para que los estudiantes tomen decisiones razonables debe establecerse un procedimiento que garantice su asesoramiento, puntualizando que no basta sólo con tener información (sobre becas o salidas profesionales), sino que ésta tiene que ir acompañada de un adecuado asesoramiento, convirtiéndose los servicios de orientación en un punto de enlace básico entre los estudiantes y la sociedad. Villena, Muñoz-García, Polo y Jiménez (2010) pusieron de manifiesto que el marco europeo contempla la acción tutorial como una parte de la actividad orientadora y como señalan Romero, Zurita y Zurita (2010), para que el estudiante pueda desarrollar un trabajo autónomo necesita las tutorías como herramientas de orientación y seguimiento.

Nuestra sociedad se caracteriza por una progresiva diversidad de opciones en las que cada persona desarrolla múltiples acciones de búsqueda y de orientación en las distintas etapas de su ciclo vital. De ahí, como afirma Pérez (2010), la comunidad educativa es el reflejo de esta sociedad plural, competitiva y exigente, y al mismo tiempo vulnerable, frágil e incluso problemática, que necesita directrices que garanticen una orientación de calidad, siendo ésta una tarea pendiente en el ámbito universitario. Además, la enseñanza en masas genera un problema que sólo se puede subsanar con la creación de servicios de apoyo u orientación, siendo éste un criterio de calidad (Álvarez, 2001).

Ante este panorama, es inevitable pensar que los cambios ocurridos en la enseñanza superior (el crecimiento del número de alumnos, el aumento de la diversidad, más posibilidades de elección, uso de tecnologías de la información y la comunicación, etc.) constituyen para la orientación a este nivel un reto fundamental (Jiménez, 2007), que debe planificarse y desarrollarse en los centros, estableciendo sus contenidos a partir de la evaluación de sus necesidades (Álvarez, 2001), motivaciones y estrategias de aprendizaje (Valentín et al., 2013).

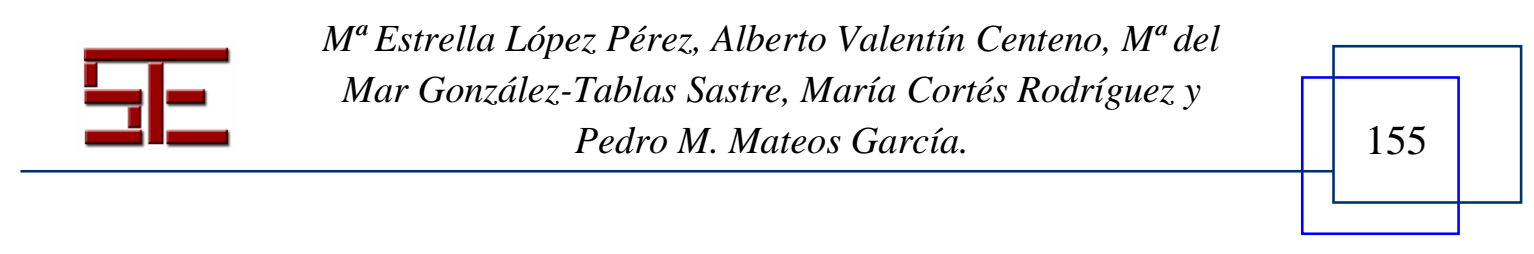




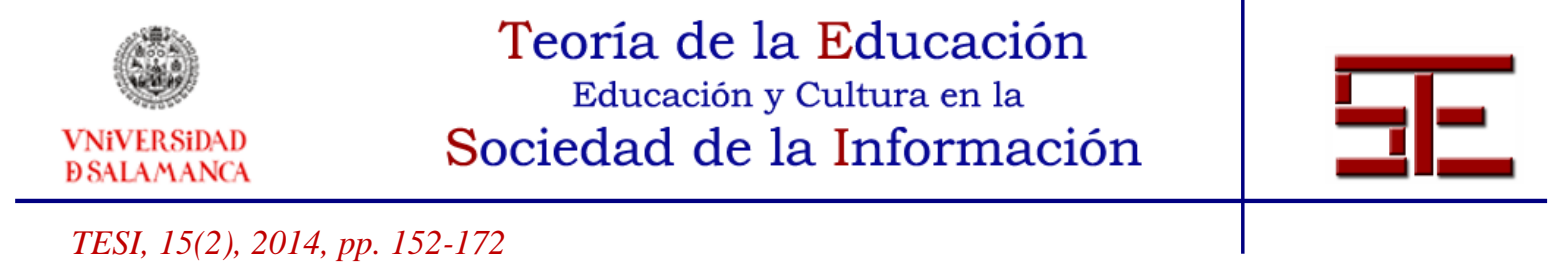

Uno de los objetivos fundamentales de la orientación en los estudios universitarios es proporcionar a los alumnos herramientas que les permitan tomar decisiones en este proceso para poder alcanzar su desarrollo vocacional. La investigación desarrollada en los últimos años por Rivas, Martín y De Asís Martín (2008), se ha centrado en conocer la conducta vocacional de los estudiantes universitarios, ya que su conocimiento permite llegar a tomar decisiones y potenciar la actividad mental, el compromiso y la significación personal. Rivas, Tormos y Martínez (2005), en un intento de lograr un indicador eficaz en la discriminación de la conducta vocacional de los estudiantes universitarios, analizaron en profundidad la información ofrecida por los BIODATOS. Con el fin de evaluar esta información, que tradicionalmente se trata como "datos de identificación", crearon el Cuestionario de Biodatos Universitarios (CBU) (Rocabert, 2005). Para su elaboración se centraron en los Biodatos académicos, que como afirman Rocabert, Descals y Gómez (2007) son "los que parecen mostrar una mayor relación con los intereses y las elecciones vocacionales de los estudiantes" (p. 18). Dicha elaboración requirió analizar diferentes aspectos relativos al desarrollo vocacional de 2169 universitarios de diferentes titulaciones, con el fin de averiguar cuáles son las opciones de especialización y de preparación laboral que pueden determinar el grado de profesionalidad futura. Ello les permitió describir los siguientes factores o indicadores de Biodatos: Motivación o Interés por la opción universitaria escogida, Toma de Decisiones Académicas o Preprofesionales, Grado de Decisión sobre la especialidad o salida profesional, Experiencia Laboral y Dificultades percibidas en el proceso de Toma de Decisiones Preprofesionales.

Por otro lado, la tutorización del Practicum de Psicología de la Universidad de Salamanca (USAL) nos ha permitido detectar la necesidad que tienen nuestros estudiantes de recursos que les permitan desarrollar su vocación (López; Valentín \& González-Tablas, 2012). El Prácticum como experiencia reduce la distancia que separa el mundo académico de la futura actividad profesional (Bausela, 2012). Siguiendo a Cid, Pérez y Sarmiento (2011) los alumnos que cursan esta asignatura encuentran en sus tutorías apoyo para su desarrollo profesional y para su socialización, además de ayudarles a realizar los aprendizajes relacionados con la experiencia del contacto directo con el mundo laboral. Sin embargo, este recurso está encaminado a orientar sólo durante el proceso formativo en la práctica pre-profesional (Martínez \& Raposo, 2011), pero no durante la programación de los estudios o las opciones de especialización.

Considerando todo lo anterior, hemos de reconocer que cada titulación tiene unos intereses y características específicas y por ello los servicios de orientación deben adaptarse a los mismos. En consecuencia, el objetivo del presente trabajo consiste en analizar los indicadores de la conducta vocacional de los estudiantes universitarios de Psicología, su evolución a lo largo de su formación académica y su comparación con datos de otras poblaciones universitarias.

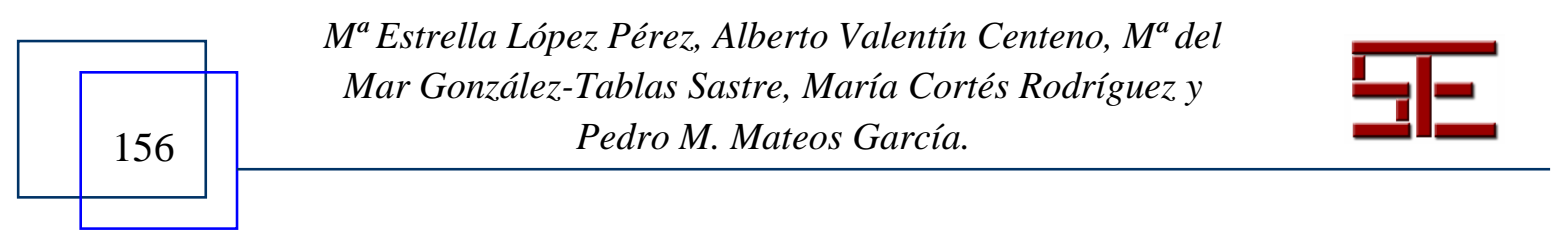




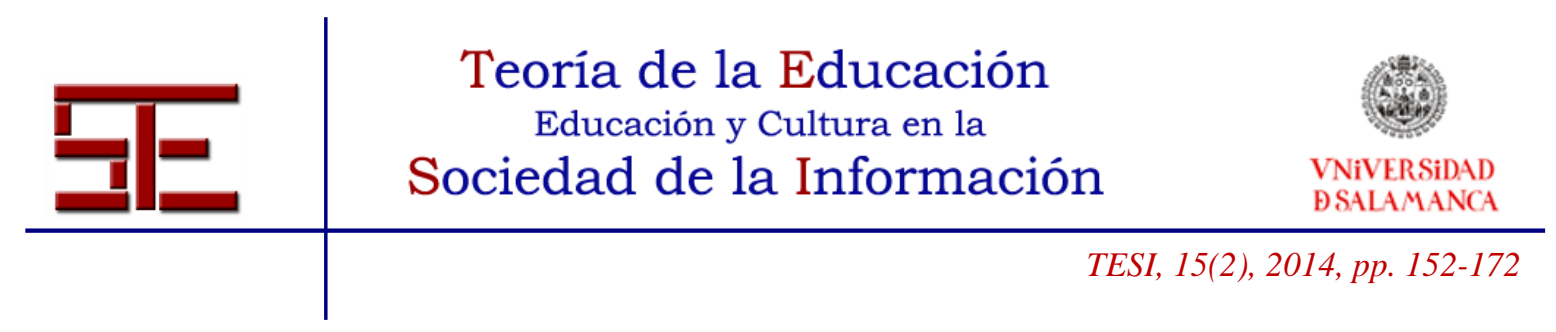

\section{2.- METODOLOGÍA}

\section{1.- Muestra}

Participaron en el estudio 329 estudiantes de la Facultad de Psicología de la USAL, 248 alumnos de segundo curso (32 varones) y 81 de quinto (10 varones). La desproporción entre varones y mujeres en la muestra es reflejo de la desigual distribución por género que aparece entre los estudiantes de dicha titulación. Los datos fueron recogidos durante el mes de diciembre de 2010.

\section{2.- Instrumentos}

Para realizar el estudio se seleccionó como instrumento de recogida de datos el CBU de Rocabert (2005). El cuestionario abarca cinco indicadores académicos sobre las conductas realizadas por los estudiantes en relación a su planificación vocacional y preprofesional, a saber: la Motivación o Interés por la opción universitaria escogida, la Toma de Decisiones académicas o preprofesionales (asignaturas optativas, Practicum), el Grado de Decisión sobre la especialidad o salida profesional, la Experiencia Laboral y las Dificultades Percibidas en el proceso de toma de decisiones preprofesionales. Las instrucciones requieren que el alumno, en cada uno de los 9 ítems del cuestionario, seleccione la opción que mejor defina su situación, pudiendo elegir más de una alternativa de respuesta. Rocabert et al. (2007) analizaron la calidad psicométrica del cuestionario informando que los ítems son homogéneos y buenos indicadores de la conducta vocacional.

\section{3.- Procedimiento}

El cuestionario fue administrado de manera colectiva al finalizar una clase, en la que se solicitó a los alumnos su participación voluntaria. El tiempo empleado para su cumplimentación no superó los 15 minutos y aunque la prueba no era anónima, se aseguró la estricta confidencialidad de la misma. Para el análisis de los datos recogidos se utilizó el paquete estadístico SPSS versión 18.0 para Windows. Se realizaron pruebas de comparación de muestras utilizando la técnica U de Mann-Whitney, que detecta diferencias entre dos grupos independientes. Es una de las pruebas no paramétricas más poderosas y constituye una buena alternativa al contraste $t$ de diferencia de medias (Siegel, 1970). Además, para medir la asociación entre conjuntos y atributos se utilizó el Análisis de Contingencia que facilita el paquete estadístico SPSS.

\section{3.- RESULTADOS Y DISCUSIÓN}

Para conocer las vivencias, hechos y conductas de los estudiantes en relación a la evolución de su desarrollo vocacional, así como su eficacia a la hora de seleccionar las opciones para su futuro profesional, se realizó un análisis siguiendo los indicadores de Biodatos aportados por Rocabert et al. (2007). Estos autores elaboraron el cuestionario CBU con el fin de analizar los datos biográficos dentro del área académica relacionados

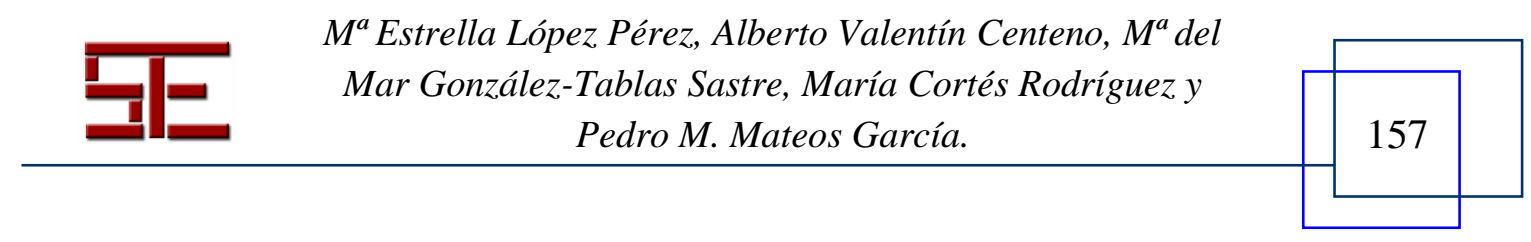




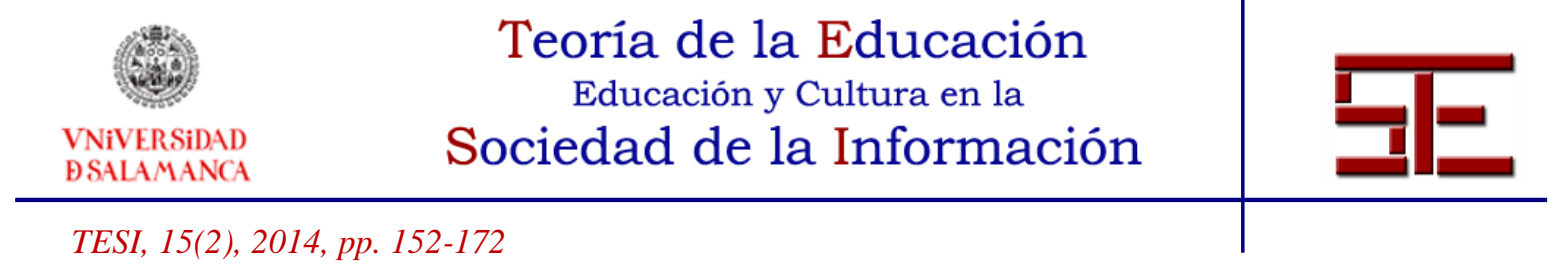

con el desarrollo vocacional de los estudiantes. Con una muestra de 2.169 estudiantes de último curso de doce universidades españolas, los resultados del estudio realizado por estos autores mostraron que los datos del cuestionario permitían predecir el adecuado desarrollo de carrera de los estudiantes. A continuación se muestran los resultados a partir del análisis de cada uno de los indicadores recogidos en el cuestionario propuesto por Rocabert et al. (2007).

\section{1.- Motivación o Interés por la opción universitaria escogida}

El primer indicador está formado por los ítems 1, 2 y 3. Entre estos ítems, el primero pregunta a los estudiantes por el orden que ocupaban los estudios que están realizando entre sus preferencias. La mayoría de los participantes afirmaron que pudieron hacer los estudios elegidos como primera opción (89,4\%). Según la prueba U de Mann-Whitney transformada a $\mathrm{z}$, no se detectaron diferencias significativas entre los alumnos de segundo y de quinto curso $(\mathrm{zu}=-0,689 ; \mathrm{p}>0,245)$. Este resultado es consistente con el obtenido por Rocabert et al. (2007), donde el $78 \%$ de los alumnos matriculados lo habían hecho en los estudios elegidos, y también con el que recoge el informe emitido por la Universidad Politécnica de Madrid sobre el perfil de los alumnos de nuevo ingreso (Gabinete de Estudios Psicológicos y Estadística, 2006).

El segundo ítem, Trayectoria Evolutiva del Interés por los Estudios, pregunta al estudiante en qué periodo de su vida se planteó por primera vez estudiar la carrera que está cursando. Los resultados indican que un 50,18\% de los alumnos que estudian Psicología empezaron a estar interesados en esta titulación durante el Bachillerato y un 43,8\% mostraron su interés en edades más tempranas (12-16 años), periodo que corresponde a la Educación Secundaria Obligatoria. Por lo que se refiere a la evolución, no se detectaron diferencias entre los dos cursos sobre el periodo en el que comenzaron a mostrar su interés por la psicología $(z u=0,119 ; \mathrm{p}>0,905)$.

Los datos obtenidos sobre cuándo se plantearon por primera vez cursar los estudios de Psicología contrastan con los resultados obtenidos por Rocabert et al. (2007), donde los estudiantes $(62,6 \%)$ afirmaban haber elegido sus estudios universitarios más tarde (entre los 17 y 18 años). Esta diferencia puede ser debida a la heterogeneidad de estudios analizados por estos autores frente a lo específico de nuestra muestra. Tengamos en cuenta que la Psicología puede implicar un aspecto vocacional de carácter solidario y humanista en cualquiera de sus perfiles profesionales, como ocurre en otras ciencias médicas como la medicina o la enfermería (Ledo; Fernández, 2009) y que suele despertarse en edades tempranas. Según Rivas (2003), estos estudios estarían dentro del grupo Vocacional Biosanitario, donde entre sus rasgos generales podemos encontrar el interés por las relaciones personales, la salud y ayudar a la gente.

Desde una perspectiva centrada en la optimización humana como meta de crecimiento personal, a la edad en que los estudiantes eligieron Psicología como carrera, o profesión,

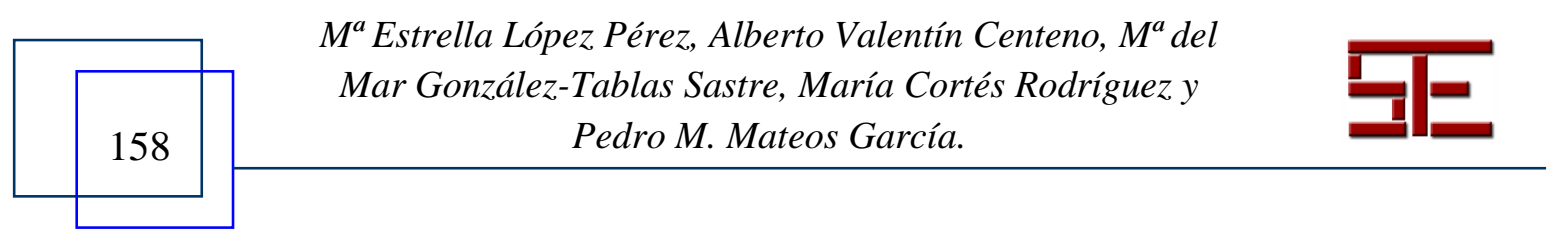




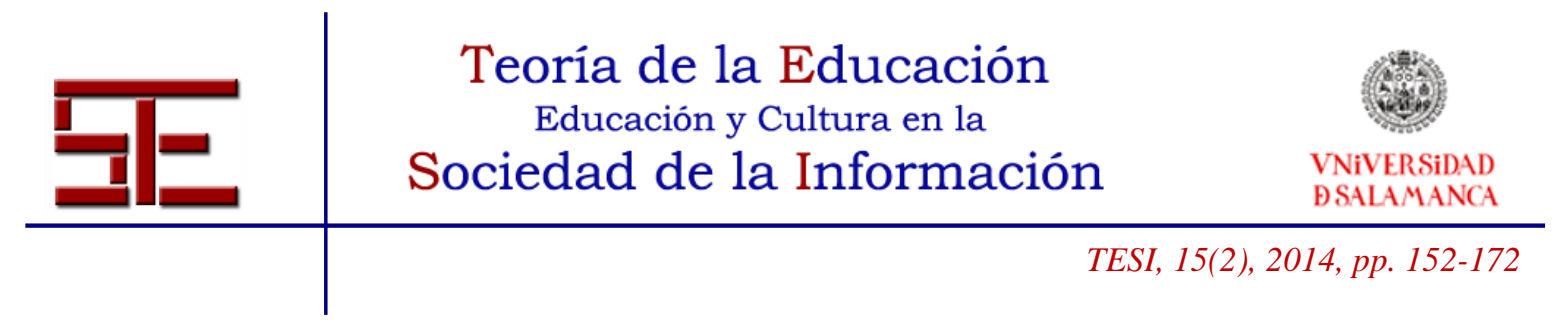

se encuentran proyectando sus objetos-fines. Ante esta necesidad de definir su futuro, la trayectoria evolutiva permite una búsqueda más eficaz y constructiva (Cárcamo, Contreras, Muñoz \& Nesbet, 2008). En este periodo, dado que la opción vocacional ya está decidida, el papel de los servicios de orientación debería centrarse en informar sobre las ofertas académicas y los planes de estudios relacionados con la opción elegida.

Al analizar el ítem 3 (Tabla 1), Grado de satisfacción con la Carrera, que refleja el agrado con los estudios elegidos, se encontró que la mayoría de los estudiantes de Psicología se muestran muy satisfechos $(52,6 \%)$ o satisfechos $(38,3 \%)$. Esto indica que un $90 \%$ de los alumnos se sienten complacidos con su elección.

Tabla 1.

Tabla de frecuencias y porcentajes de Satisfacción con la Carrera

\begin{tabular}{lcccccc}
\hline \multirow{2}{*}{ Satisfacción } & \multicolumn{2}{c}{ Segundo } & \multicolumn{2}{c}{ Quinto } & \multicolumn{2}{c}{ Total } \\
\cline { 2 - 7 } & $\mathbf{N}$ & $\mathbf{\%}$ & $\mathbf{N}$ & $\mathbf{\%}$ & $\mathbf{N}$ & $\mathbf{\%}$ \\
\hline Insatisfecho & 1 & 0,4 & 3 & 3,7 & 4 & 1,2 \\
Intermedio & 15 & 6,0 & 11 & 13,6 & 26 & 7,9 \\
Satisfecho & 94 & 37,9 & 32 & 39,5 & 126 & 38,3 \\
Muy Satisfecho & 138 & 55,6 & 35 & 43,2 & 173 & 52,6 \\
\hline \multicolumn{1}{c}{ Total } & 248 & 100 & 81 & 100 & 329 & 100 \\
\hline
\end{tabular}

Además, se detectaron diferencias significativas entre los dos cursos (U de MannWhitney; $\mathrm{zu}=-2,512 ; \mathrm{p}<0,006$, unilateral), mostrándose más satisfechos los de segundo. La visión más pesimista de los alumnos de quinto puede ser debida a la finalización inminente de sus estudios, unida a la incertidumbre sobre su futuro profesional.

Salanova, Martínez, Bresó, Llorens y Grau (2005) estudiaron el bienestar psicológico de los estudiantes de la Universitat Jaume I, encontrando que, en general, existen buenos niveles de satisfacción con los estudios. Los alumnos de los cursos superiores también presentaban menor compromiso y satisfacción de forma consistente con nuestros resultados. Salanova et al. (2005) interpretan este resultado como una consecuencia de la ausencia de cumplimiento de las expectativas que sucede a lo largo del proceso de socialización del estudiante en la Universidad.

\section{2.- Toma de Decisiones Académicas o Preprofesionales}

El segundo indicador lo configuran los ítems 4 y 5, relacionados con las asignaturas optativas y el Practicum. En relación al ítem 4, Elección de Asignaturas Optativas, analizamos los criterios que tuvieron en cuenta los estudiantes a la hora de seleccionar estas materias (Tabla 2). En este ítem se proponían 7 opciones de respuesta y los participantes podían señalar tantas como consideraran oportuno.

$M^{a}$ Estrella López Pérez, Alberto Valentín Centeno, $M^{a}$ del Mar González-Tablas Sastre, María Cortés Rodríguez y

Pedro M. Mateos García. 


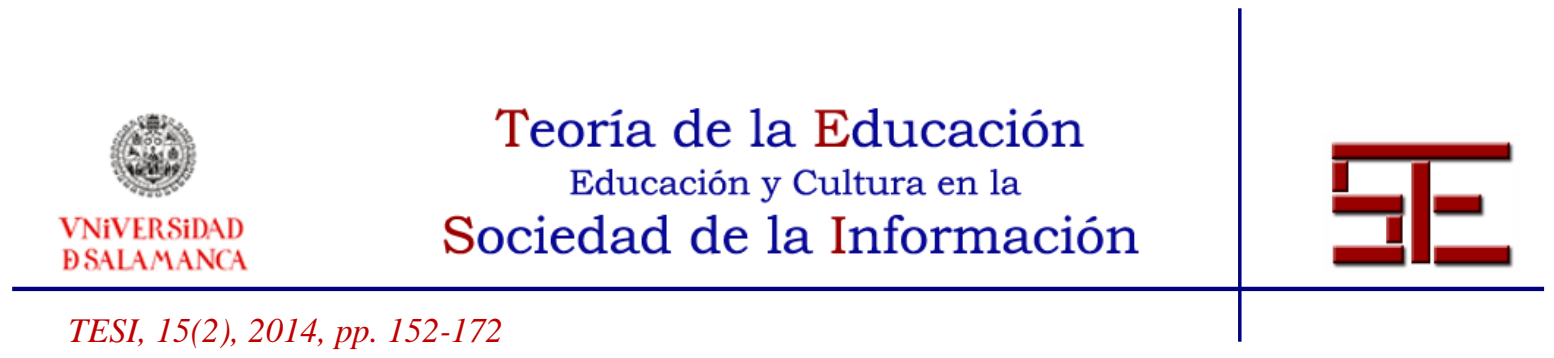

Tabla 2

Tabla de Frecuencias y porcentajes de Elección de las Asignaturas Optativas

\begin{tabular}{|c|c|c|c|c|c|c|c|c|}
\hline \multirow{3}{*}{$\begin{array}{c}\text { Elección de } \\
\text { Optativas }\end{array}$} & \multirow{3}{*}{$\begin{array}{c}\text { Participantes } \\
\text { Total(N=329) } \\
\%\end{array}$} & \multicolumn{4}{|c|}{ Respuestas } & \multirow{2}{*}{\multicolumn{2}{|c|}{ Total }} & \multirow{3}{*}{$\mathbf{Z}_{\mathrm{u}} ; \mathbf{p}$} \\
\hline & & \multicolumn{2}{|c|}{ Segundo } & \multicolumn{2}{|c|}{ Quinto } & & & \\
\hline & & $\mathbf{N}$ & $\%$ & $\mathbf{N}$ & $\%$ & $\mathbf{N}$ & $\%$ & \\
\hline $\begin{array}{l}\text { Relación con la } \\
\text { especialidad }\end{array}$ & 54,3 & 122 & 27,1 & 56 & 25,5 & 178 & 26,6 & $\begin{array}{c}-3,122 \\
p<.001\end{array}$ \\
\hline $\begin{array}{l}\text { Elección de tus } \\
\text { compañeros }\end{array}$ & 4,0 & 7 & 1,6 & 6 & 2,7 & 13 & 1,9 & $\begin{array}{l}-1,836 \\
p \leq .033\end{array}$ \\
\hline $\begin{array}{l}\text { No existe } \\
\text { optatividad }\end{array}$ & 6,4 & 21 & 4,7 & 0 & 0,0 & 21 & 3,1 & $\begin{array}{l}-2,703 \\
p \leq .003\end{array}$ \\
\hline Horario & 32,0 & 62 & 13,8 & 43 & 19,5 & 105 & 15,7 & $\begin{array}{l}-4,701 \\
p<.001\end{array}$ \\
\hline Profesor/a & 12,5 & 17 & 3,8 & 24 & 10,9 & 41 & 6,1 & $\begin{array}{l}-5,380 \\
p<.001\end{array}$ \\
\hline $\begin{array}{l}\text { Facilidad de la } \\
\text { materia }\end{array}$ & 25,3 & 53 & 11,8 & 30 & 13,6 & 83 & 12,4 & $\begin{array}{l}-2,814 \\
p \leq .002\end{array}$ \\
\hline $\begin{array}{l}\text { Interés por los } \\
\text { contenidos }\end{array}$ & 69,8 & 168 & 37,3 & 61 & 27,7 & 229 & 34,2 & $\begin{array}{l}-1,283 \\
p \leq .099 \\
\end{array}$ \\
\hline \multicolumn{2}{|c|}{ Total respuestas } & 450 & 100 & 220 & 100 & 670 & 100 & \\
\hline
\end{tabular}

Los participantes encuestados emitieron 670 respuestas mostrando que, en general, el criterio más importante a la hora de elegir estas asignaturas fue el interés por los contenidos, respuesta elegida por el $69,8 \%$ de los estudiantes, seguido por la relación con la especialidad o trabajo futuro $(54,3 \%)$. Estos resultados coinciden con los obtenidos por Rocabert et al. (2007), lo cual sugiere que estos aspectos son los más importantes para los estudiantes a la hora de tomar decisiones académicas, independientemente de la titulación y de la universidad en la que se encuentren.

El horario en que se impartían las diferentes asignaturas también fue apreciado como un criterio relevante por el $32 \%$ de los participantes, constituyendo el $15,7 \%$ de las respuestas emitidas. Sin embargo, llama la atención que este criterio fuera considerado más importante por los alumnos de quinto, que ya han tenido que elegir materias, que por los de segundo, que todavía no tienen asignaturas optativas (U de Mann-Whitney; $\mathrm{zu}=-4,701 ; \mathrm{p}<0,001)$. Por otro lado, es interesante descubrir que un 10,9\% de las respuestas de los alumnos de quinto indican que optan a las asignaturas por el profesor, frente a un 3,8\% de las respuestas de los de segundo. En algunos casos, los profesores tienen docencia en diferentes cursos y los alumnos de quinto ya los conocen, con lo que pueden decidir coger o no su asignatura apoyándose en esta experiencia previa.

El ítem 5, Elección del Practicum, recoge los criterios considerados por el estudiante a la hora de elegir dónde realizar las prácticas de la titulación. En este ítem se proponen 10 opciones de respuesta, de las cuales el participante puede señalar hasta un máximo de tres. Se emitieron 768 respuestas (Tabla 3), cuyo análisis indica que los alumnos de

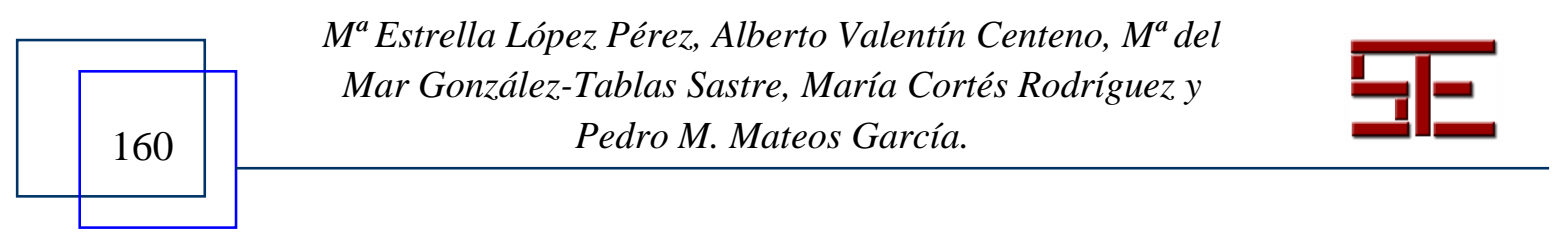




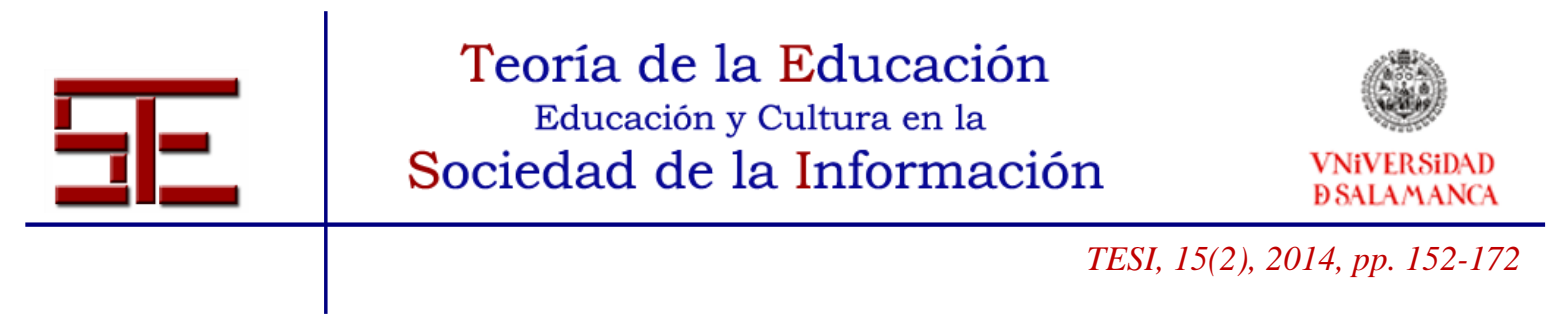

Psicología eligen sus prácticas, en primer lugar, por la especialidad a la que quieren dedicarse $(80,2 \%)$, seguido por el interés que despierta en ellos las tareas que se realizan en las prácticas $(54,6 \%) \mathrm{y}$, en tercer lugar, por las posibilidades de salidas profesionales $(51,5 \%)$.

Tabla 3.

Tabla de frecuencias y porcentajes de Elección del Practicum

\begin{tabular}{|c|c|c|c|c|c|c|c|c|}
\hline \multirow{3}{*}{$\begin{array}{l}\text { Elección del } \\
\text { Practicum }\end{array}$} & \multirow{3}{*}{$\begin{array}{c}\text { Participantes } \\
\text { Total(N=329) } \\
\%\end{array}$} & \multicolumn{4}{|c|}{ Respuestas } & \multirow{2}{*}{\multicolumn{2}{|c|}{ Total }} & \multirow{3}{*}{$\mathbf{Z}_{\mathrm{U}} ; \mathbf{p}$} \\
\hline & & \multicolumn{2}{|c|}{ Segundo } & \multicolumn{2}{|c|}{ Quinto } & & & \\
\hline & & $\mathbf{N}$ & $\%$ & $\mathbf{N}$ & $\%$ & $\mathbf{N}$ & $\%$ & \\
\hline $\begin{array}{l}\text { Horario y } \\
\text { proximidad }\end{array}$ & 6,4 & 12 & 2,1 & 9 & 4,5 & 21 & 2,7 & $\begin{array}{r}-2,002 \\
p \leq .022\end{array}$ \\
\hline $\begin{array}{l}\text { Buenas referencias } \\
\text { del centro }\end{array}$ & 21,0 & 45 & 7,9 & 24 & 12,1 & 69 & 9,0 & $\begin{array}{l}-2,201 \\
p \leq .014\end{array}$ \\
\hline $\begin{array}{l}\text { Orientación del } \\
\text { Tutor/a de prácticas }\end{array}$ & 8,5 & 23 & 4,0 & 5 & 2,5 & 28 & 3,6 & $\begin{array}{r}-0,867 \\
p \leq 193\end{array}$ \\
\hline $\begin{array}{l}\text { Posibilidades de } \\
\text { futuras salidas } \\
\text { profesionales }\end{array}$ & 51,5 & 132 & 23,2 & 37 & 18,6 & 169 & 22.0 & $\begin{array}{l}-1,178 \\
p \leq .119\end{array}$ \\
\hline $\begin{array}{l}\text { Especialidad a la } \\
\text { que quieres } \\
\text { dedicarte }\end{array}$ & 80,2 & 200 & 35,1 & 63 & 31,7 & 263 & 34,2 & $\begin{array}{r}-0,559 \\
p \leq .288\end{array}$ \\
\hline $\begin{array}{l}\text { Interés por las tareas } \\
\text { que allí se realizan }\end{array}$ & 54,6 & 123 & 21,6 & 56 & 28,1 & 179 & 23,3 & $\begin{array}{c}-3,061 \\
p<. .001\end{array}$ \\
\hline $\begin{array}{l}\text { No tuviste } \\
\text { posibilidad de } \\
\text { elección }\end{array}$ & 0,9 & 1 & 0,2 & 2 & 1,0 & 3 & 0,4 & $\begin{array}{l}-1,696 \\
p \leq .045\end{array}$ \\
\hline $\begin{array}{l}\text { En mis estudios no } \\
\text { existe practicum }\end{array}$ & 0,3 & 0 & 0,0 & 1 & 0,5 & 1 & 0,1 & $\begin{array}{l}-1,750 \\
p \leq .040\end{array}$ \\
\hline $\begin{array}{l}\text { No he pensado en } \\
\text { ello }\end{array}$ & 4,6 & 15 & 2,6 & 0 & 0.0 & 15 & 2,0 & $\begin{array}{l}-2,262 \\
p \leq .012\end{array}$ \\
\hline $\begin{array}{l}\text { No tengo claro qué } \\
\text { elegir }\end{array}$ & 6,1 & 18 & 3,2 & 2 & 1,0 & 20 & 2,6 & $\begin{array}{c}2,262 \\
p \leq 059\end{array}$ \\
\hline Total res & destas & 569 & 100 & 199 & 100 & 768 & 100 & \\
\hline
\end{tabular}

Si comparamos estos datos con los obtenidos por Rocabert et al. (2007), se observa que los criterios que tenían en cuenta los participantes de su muestra a la hora de elegir las prácticas son similares a los manifestados por los alumnos de Psicología, si bien éstos se preocupan más por adquirir experiencia en la especialidad a la que quieren dedicarse.

Los estudiantes de quinto hicieron al comienzo del curso la elección del Practicum, mientras que los de segundo todavía no han podido tomar esta decisión y contestaron de forma más "ideal", sin considerar las incompatibilidades que puedan aparecer en el momento de hacerlo. Las significaciones aportadas por la prueba U de Mann-Whitney señalan las diferencias que existen entre los dos grupos, destacando que para los

$M^{a}$ Estrella López Pérez, Alberto Valentín Centeno, $M^{a}$ del Mar González-Tablas Sastre, María Cortés Rodríguez y Pedro M. Mateos García. 


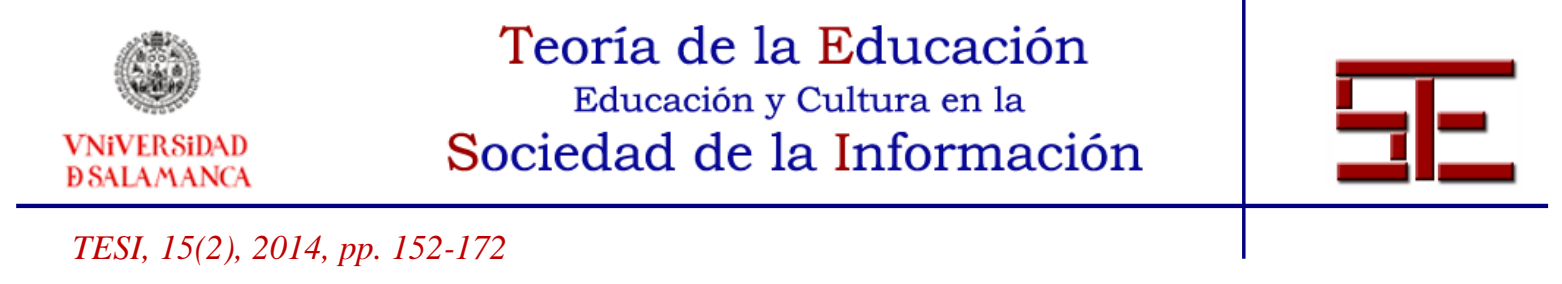

alumnos de quinto, el interés por las tareas, las buenas referencias del centro o el horario y proximidad a casa son criterios más importantes que para los de segundo.

\section{3.- Grado de Decisión sobre la especialidad o salida profesional}

El tercer indicador está formado por los ítems 6 y 8. Dicho indicador nos permite identificar a los alumnos que ya han tomado una decisión sobre la especialidad a la que quieren dedicarse, detectar a los que aún no lo han decidido, así como a aquellos que creen necesitar ayuda para tomar esta decisión. A diferencia de los anteriores ítems del cuestionario en los que las respuestas superaron de forma importante a los sujetos, en el caso del ítem 6, los 327 alumnos emitieron sólo 340 respuestas (Tabla 4). Un porcentaje relativamente pequeño de la muestra, el 7,3\%, afirmó necesitar ayuda y el 28,1\% manifestó que aún no tenían decidido nada sobre esta cuestión. El 36,1\% tenía muy claro la especialidad a la que querrían dedicarse y un $23,2 \%$ dudaba entre dos. En la comparación de estos resultados con los obtenidos por Rocabert et al. (2007) en población universitaria general, no existen grandes diferencias.

Tabla 4.

Tabla de frecuencias y porcentajes de Especialidad Profesional

\begin{tabular}{|c|c|c|c|c|c|c|c|}
\hline \multirow{2}{*}{ Especialidad Profesional } & \multicolumn{2}{|c|}{ Segundo } & \multicolumn{2}{|c|}{ Quinto } & \multicolumn{2}{|c|}{ Total } & \multirow{2}{*}{$\mathbf{Z}_{\mathrm{U}} ; \mathbf{p}$} \\
\hline & $\mathbf{N}$ & $\%$ & $\mathbf{N}$ & $\%$ & $\mathbf{N}$ & $\%$ & \\
\hline $\begin{array}{l}\text { Tengo muy claro lo que quiero } \\
\text { hacer }\end{array}$ & 86 & 35,0 & 32 & 39,5 & 118 & 36,1 & $\begin{array}{l}-0,586 \\
p \leq .558\end{array}$ \\
\hline Dudo entre dos especialidades & 58 & 23,6 & 18 & 22,2 & 76 & 23,2 & $\begin{array}{l}-0,004 \\
p \leq .997\end{array}$ \\
\hline Dudo entre tres especialidades & 23 & 9,3 & 7 & 8,6 & 30 & 9,2 & $\begin{array}{l}-0,171 \\
p \leq .864\end{array}$ \\
\hline No tengo decidido nada & 73 & 29,7 & 19 & 23,5 & 92 & 28,1 & $\begin{array}{l}-1,039 \\
p \leq .299\end{array}$ \\
\hline Necesitaría ayuda & 10 & 4,1 & 14 & 17,3 & 24 & 7,3 & $\begin{array}{l}-3,976 \\
p<.000 \\
\end{array}$ \\
\hline Total & 250 & 100 & 90 & 100 & 340 & 100 & \\
\hline
\end{tabular}

Por otra parte, la proporción de alumnos de quinto que afirmaron necesitar ayuda fue mayor que la de los de segundo. Esto puede ser debido a que los alumnos de quinto ven próximo el final de sus estudios y esta situación provoca que aumente la incertidumbre sobre su futuro inmediato. Por el contrario, los estudiantes de segundo curso pueden creer que van a adquirir los conocimientos necesarios para elegir especialidad en los años de carrera que aún les quedan. En general, aquellos que hicieron dos elecciones en este ítem eran los mismos alumnos que no tenían tomada una decisión y necesitaban ayuda.

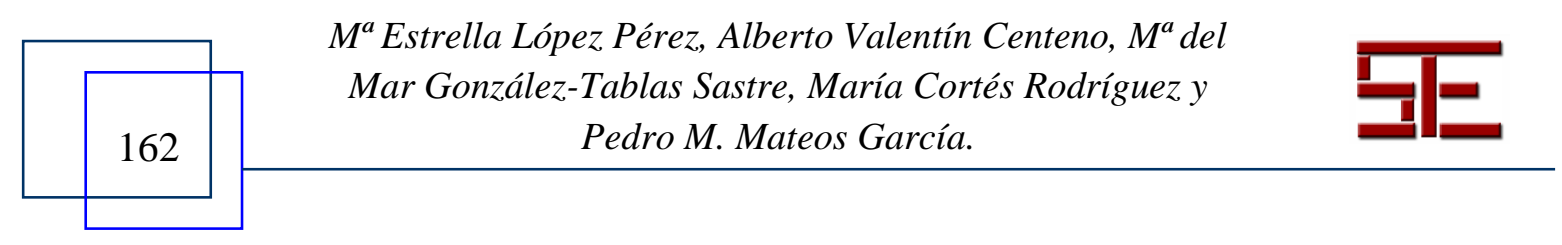




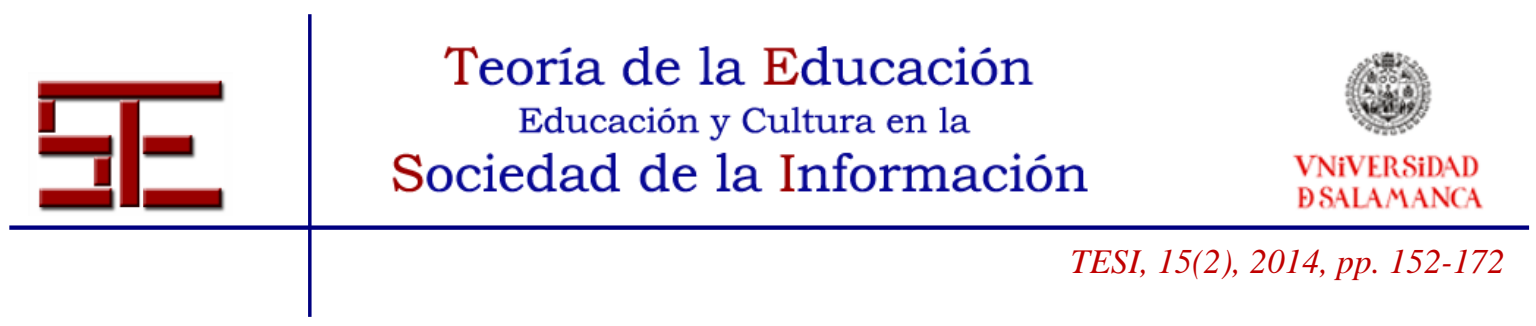

Dentro del grupo de alumnos que seleccionaron la opción "no tengo decidido nada", el 23,5\% corresponde a los alumnos de quinto curso. La experiencia del Practicum, que la mayoría todavía no han realizado, en ocasiones aclara este aspecto a nuestros estudiantes, ya que al entrar en contacto con el mundo profesional confirman sus preferencias sobre la especialidad a la que quieren dedicarse y también conocen las vías de acceso al mismo. Según Cid et al. (2011) uno de los beneficios de las tutorías de esta asignatura es precisamente el apoyo para el desarrollo profesional de nuestros alumnos, además de la ayuda para realizar aprendizajes de forma reflexiva y la socialización.

Además, el ítem 6, al incorporar un formato de respuestas abiertas, nos permite identificar posibles demandas de especialización. Las elecciones de los alumnos se agruparon en función de las especialidades a las que se adscriben las salidas profesionales de la Psicología: Psicología Clínica y de la Salud, Psicología Evolutiva y de la Educación, y por último, Psicología Social y de las Organizaciones. Se incluyeron en la categoría "otras" aquellas opciones elegidas por los alumnos no clasificables en éstas grandes áreas. En relación con las especialidades por las que sienten más interés los alumnos, la opción de Psicología Clínica y de la Salud es la preferida por el 49,6\% como primera y única opción. La segunda especialidad por la que muestran preferencia un elevado porcentaje de alumnos $(32,8 \%)$ es la Psicología Social y de las Organizaciones, mientras que otra área clásica, la Psicología Evolutiva y de la Educación, es elegida como especialidad por un número reducido de estudiantes $(10,4 \%)$, en comparación con las anteriores.

Los alumnos que dudaban entre dos especialidades $(21,1 \%)$ siguen prefiriendo como posible especialidad la Psicología Clínica y de la Salud (Tabla 5), combinada con la Psicología Social y de las Organizaciones. De nuevo, pocos alumnos tenían en cuenta la Psicología Evolutiva como posible opción de especialidad. En esta línea, llama la atención que la Gerontología, siendo una especialidad de futuro debido al envejecimiento de la población de nuestro país, apenas es tenida en cuenta por estos alumnos. Igual ocurre con la Investigación y la Docencia dentro de las distintas áreas, que no han sido consideradas como especialidades por ningún encuestado.

Tabla 5.

Tabla de Contingencia Opciones entre las que dudo-curso

\begin{tabular}{|c|c|c|c|c|c|c|}
\hline \multirow{5}{*}{ 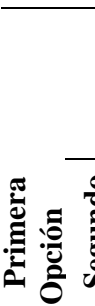 } & \multirow[b]{2}{*}{ Dudo entre dos opciones } & \multicolumn{5}{|c|}{ Segunda opción } \\
\hline & & $\begin{array}{l}\text { Clínica y } \\
\text { Salud }\end{array}$ & $\begin{array}{c}\text { Social y de las } \\
\text { Organizaciones }\end{array}$ & $\begin{array}{l}\text { Evolutiva y de } \\
\text { la Educación }\end{array}$ & Otras & Total \\
\hline & Clínica y Salud & 0,0 & 71,2 & 5,8 & 1,9 & 78,8 \\
\hline & Social y de las Organizaciones & 13,5 & 0,0 & 0,0 & 0,0 & 13,5 \\
\hline & Evolutiva y de la Educación & 5,8 & 0,0 & 0,0 & 0,0 & 5,8 \\
\hline & Otras & 1,9 & 0,0 & 0,0 & 0,0 & 1,9 \\
\hline
\end{tabular}

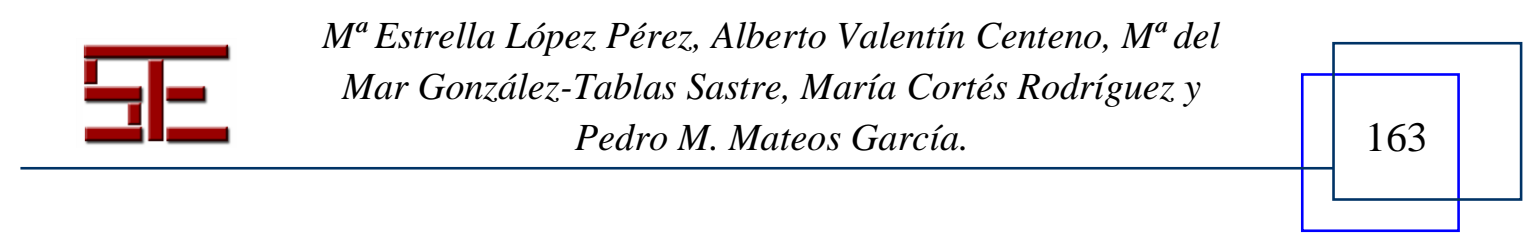




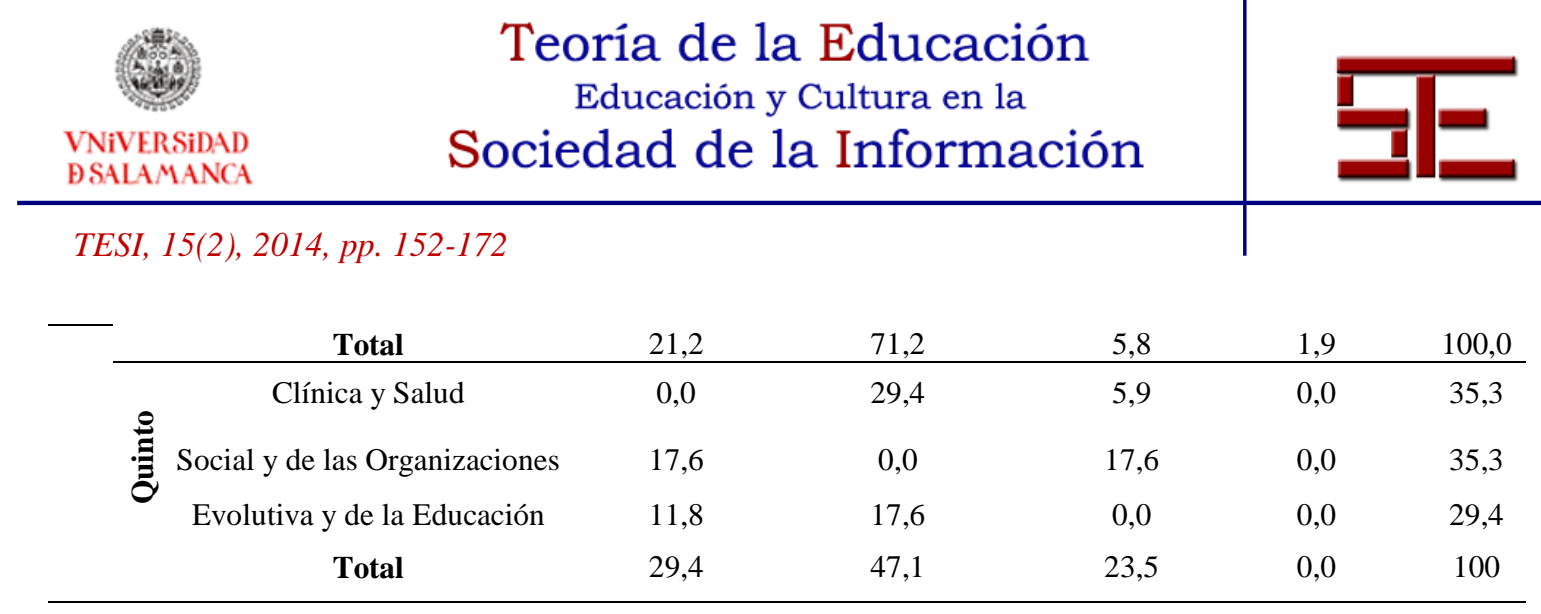

El análisis del ítem 8 refleja lo que los estudiantes tienen previsto hacer al finalizar sus estudios en Psicología (Tabla 6). A corto plazo, se planteaban inscribirse en un Máster o Postgrado $(34,8 \%)$, o bien, prepararse unas oposiciones $(19,1 \%)$, independientemente del curso en el que estuvieran matriculados. Estos resultados están en consonancia con el ítem 6, en el cual eligieron mayoritariamente la especialidad de Psicología Clínica y de la Salud, ya que ésta precisa una formación específica y el PIR como requisitos, casi obligados, para su ejercicio.

Tabla 6.

Tabla de frecuencias y porcentajes de Decisión al finalizar sus Estudios

\begin{tabular}{|c|c|c|c|c|c|c|c|c|}
\hline \multirow{3}{*}{$\begin{array}{c}\text { Decisión al } \\
\text { finalizar los estudios }\end{array}$} & \multirow{3}{*}{$\begin{array}{c}\begin{array}{c}\text { Participantes } \\
\text { Total }(\mathbf{N}=329)\end{array} \\
\%\end{array}$} & \multicolumn{6}{|c|}{ Respuestas } & \multirow{3}{*}{$\mathbf{Z}_{\mathbf{U}} ; \mathbf{p}$} \\
\hline & & \multicolumn{2}{|c|}{ Segundo } & \multicolumn{2}{|c|}{ Quinto } & \multicolumn{2}{|c|}{ Total } & \\
\hline & & $\mathbf{N}$ & $\%$ & $\mathbf{N}$ & $\%$ & $\mathbf{N}$ & $\%$ & \\
\hline Ejercer la Profesión & 20,7 & 55 & 12,2 & 13 & 8,4 & 68 & 11,3 & $\begin{array}{l}-1,181 \\
\mathrm{p} \leq 119\end{array}$ \\
\hline Solicitar una beca & 15,5 & 29 & 6,5 & 22 & 14,3 & 51 & 8,5 & $\begin{array}{l}-3,334 \\
\mathrm{p}<, 001\end{array}$ \\
\hline No lo he decidido & 19,5 & 51 & 11,4 & 13 & 8,4 & 64 & 10,6 & $\begin{array}{l}-0,890 \\
\mathrm{p} \leq 186\end{array}$ \\
\hline $\begin{array}{l}\text { Hacer un máster o } \\
\text { curso de posgrado }\end{array}$ & 64,0 & 159 & 35,4 & 51 & 33,1 & 210 & 34,8 & $\begin{array}{l}-0,187 \\
\mathrm{p} \leq 426\end{array}$ \\
\hline Hacer oposiciones & 35,1 & 82 & 18,3 & 33 & 21,4 & 115 & 19,1 & $\begin{array}{l}-1,256 \\
\mathrm{p} \leq 104\end{array}$ \\
\hline Otra carrera & 9,8 & 23 & 5,1 & 9 & 5,8 & 32 & 5,3 & $\begin{array}{l}-0,484 \\
\mathrm{p} \leq 314\end{array}$ \\
\hline Hacer el doctorado & 8,8 & 26 & 5,8 & 3 & 1,9 & 29 & 4,8 & $\begin{array}{l}-1,866 \\
\mathrm{p} \leq 032\end{array}$ \\
\hline $\begin{array}{l}\text { Buscar un trabajo } \\
\text { cualquiera }\end{array}$ & 5,8 & 11 & 2,4 & 8 & 5,2 & 19 & 3,2 & $\begin{array}{l}-1,820 \\
\mathrm{p} \leq 034\end{array}$ \\
\hline No he pensado en ello & 4,6 & 13 & 2,9 & 2 & 1,3 & 15 & 2,5 & $\begin{array}{l}-1,037 \\
p \leq 150\end{array}$ \\
\hline Total re & $\operatorname{tas}$ & 449 & 100 & 154 & 100 & 603 & 100 & \\
\hline
\end{tabular}

Por otro lado, sólo el 11,3\% de las respuestas emitidas indican que los estudiantes han decidido ejercer la profesión nada más terminar la carrera y el 4,8\% de las mismas muestran que tienen decidido hacer el doctorado, siendo más los alumnos de segundo quienes han elegido estas opciones. En sentido contrario, un mayor porcentaje de los

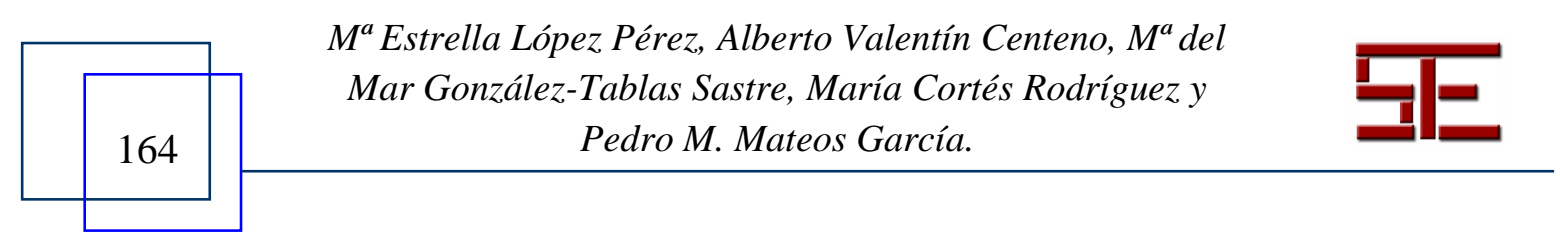




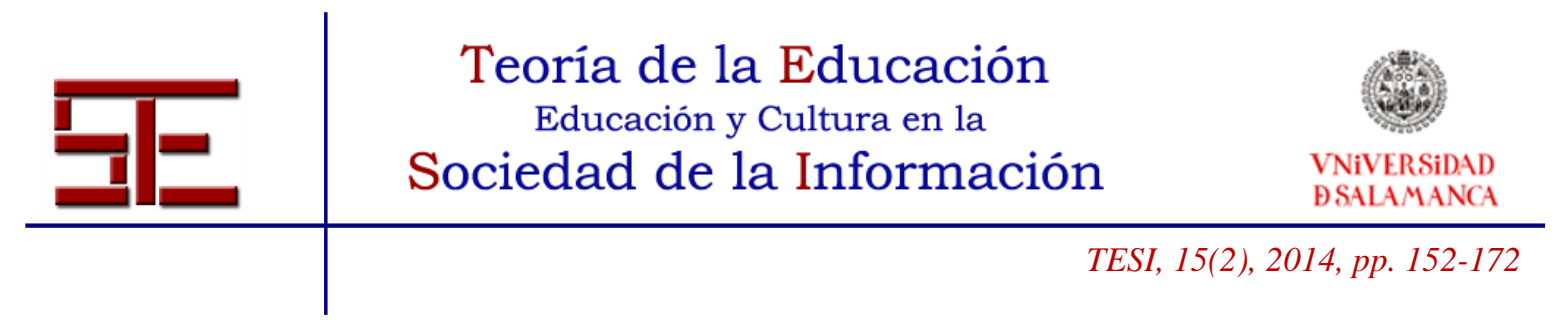

alumnos de quinto al terminar sus estudios piensan buscar un trabajo cualquiera $(5,2 \%)$. Estos datos pueden reflejar que las expectativas positivas de encontrar trabajo como psicólogo o especializarse a través de un doctorado se desvanecen cuando la incorporación al mundo laboral es inmediata.

Si comparamos estos resultados con los encontrados en el trabajo de Rocabert (2005), se distinguen dos mundos laborales o dos contextos sociales diferentes, porque el $48,8 \%$ de su muestra habían decidido ejercer la profesión. Varios factores pueden estar influyendo en esta diferencia, por un lado, su muestra fue recogida entre estudiantes de último curso procedentes de distintas titulaciones, con un porcentaje de inserción profesional quizás más elevado que el de la titulación de Psicología. Además, la recogida de datos de su estudio se realizó en el primer trimestre del año 2005, antes de la actual crisis económica que hace difícil la inserción laboral de nuestros jóvenes, traduciéndose en una preocupación de los universitarios (Vázquez \& Manassero, 2009).

La diferencia entre la proporción de elección realizada por los alumnos de segundo y quinto fue significativa, mostrando que los de quinto tienen previsto solicitar una beca para sus estudios de postgrado en mayor porcentaje que los de segundo (U de MannWhitney; $\mathrm{zu}=-3,334 ; \mathrm{p}<0,001)$.

\section{4.- Experiencia Laboral pasada o presente}

El cuarto indicador está formado por el ítem 7, y proporciona información sobre el trabajo remunerado, actual o pasado, de los alumnos. El 46,4\% de los participantes de segundo curso y el 30,9\% de quinto afirmaron no haber trabajado nunca ( $<<0,01)$. Por otro lado, dentro del grupo de estudiantes que trabajan, independientemente del curso, sólo el 4\% manifestaron realizarlo en algo relacionado con la Psicología. Estos datos difieren de los encontrados por Rocabert et al. (2007), ya que un 13,7\% de los alumnos universitarios estudiados por estos autores trabajaban en algo relacionado con sus estudios. Estas diferencias pueden ser debidas, una vez más, a que la muestra de este estudio pertenece a una única carrera mientras que en la suya se incluyeron diversos estudios superiores.

\section{5.- Dificultades percibidas en el Proceso de Toma de Decisiones}

El último indicador recoge las respuestas que aglutina el ítem 9 del cuestionario. Informa sobre cuáles son los principales escollos con los que se encuentran los encuestados para poder definirse sobre su futuro profesional. El ítem contenía 10 opciones de respuestas y los participantes podían señalar tantas como correspondieran a su caso, por lo que 329 alumnos emitieron 620 respuestas (Tabla 7).

La principal dificultad, independientemente del curso, fue la falta de información sobre las características de la especialidad en la que estaban interesados $(49,8 \%)$ y el desconocimiento sobre cuál puede ser la opción más conveniente para ellos $(38,3 \%)$. En el mismo sentido, sólo el 4,3\% de los alumnos afirmaron tener la información

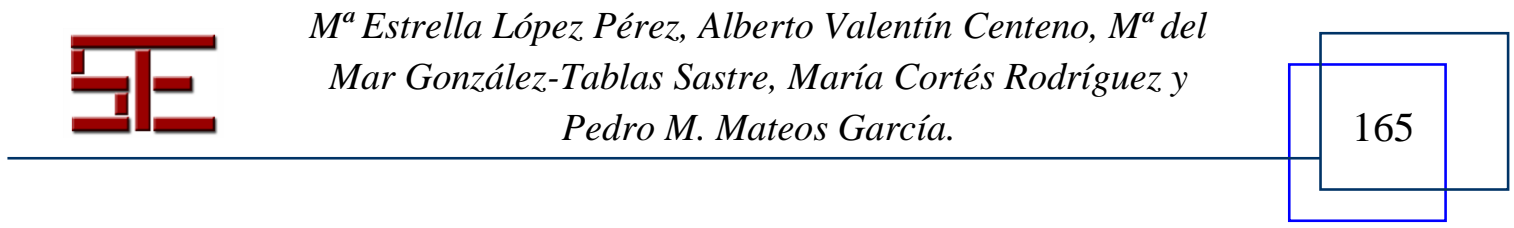




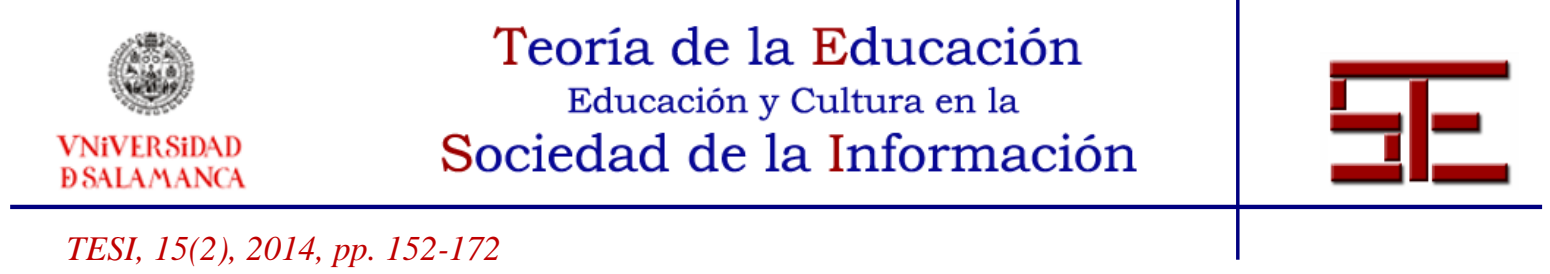

académico profesional que les hace falta. En conjunto, resulta coherente que la falta de información sobre las especialidades profesionales en Psicología, reflejada en dos de los ítems anteriores, aparezca asociada al hecho de no tener clara la opción más les conviene.

Estos resultados difieren de los publicados por Rocabert et al. (2007), donde el 26,7\%, un porcentaje notablemente inferior al obtenido por nosotros, creía que le faltaba información sobre las características de las especialidades. Además, el 27,7\% de su muestra afirmó tener la información académico-profesional que le hacía falta para tomar una decisión, frente al 4,3\% relativo a nuestros estudiantes. Ambas diferencias podrían interpretarse en el sentido de que los alumnos del estudio de Rocabert et al. (2007) sí pudieron contar con el asesoramiento necesario durante su trayectoria académica.

El análisis de las respuestas emitidas al ítem 9 muestra diferencias significativas entre los dos cursos en dos de los ítems planteados. Los alumnos de quinto eligen más las respuestas "ninguna opción me satisface plenamente" $(\mathrm{p} \leq 0,001)$ y "no tengo claro la opción que más me conviene" ( $\mathrm{p} \leq 0,004)$ que los de segundo. Una vez más, estos resultados reflejan los problemas y las dificultades percibidas por los estudiantes de último curso de carrera ante la inminente necesidad de elegir una especialidad o salida profesional.

Tabla 7.

Tabla de frecuencias y porcentajes de Dificultades para Decidirte por una Especialidad

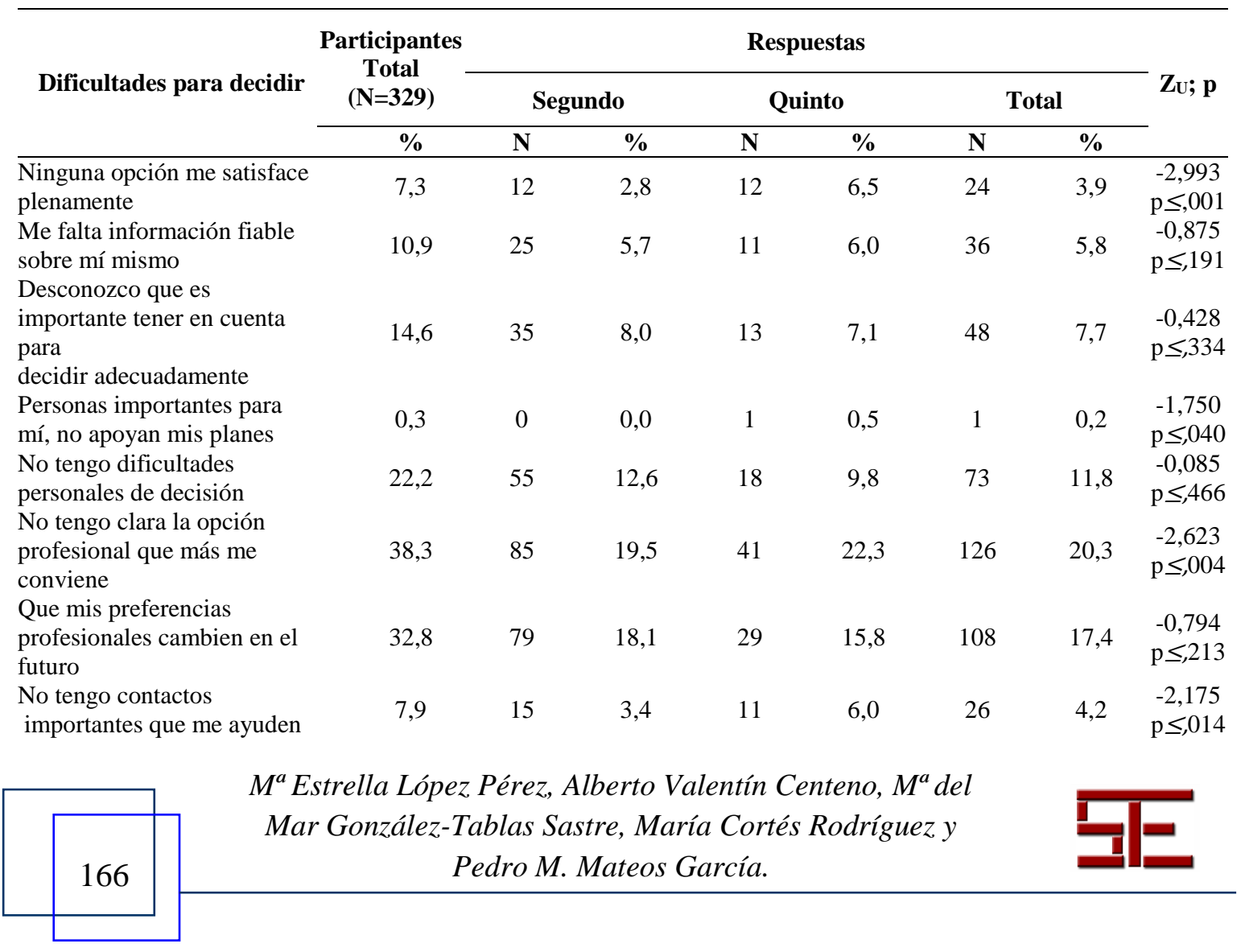




\begin{tabular}{|c|c|c|c|c|c|c|c|c|}
\hline 드 & $\begin{array}{r}\text { Teo } \\
\text { Sociec }\end{array}$ & $\begin{array}{l}d \epsilon \\
\operatorname{acio} \\
d\end{array}$ & $\begin{array}{l}\text { Ed } \\
\text { Cult } \\
\text { In }\end{array}$ & $\begin{array}{l}\text { ac } \\
\text { en } 1 \\
\mathrm{~m}\end{array}$ & & & $\begin{array}{l}\text { VNiVE } \\
\text { BSALA }\end{array}$ & $\begin{array}{l}\text { SiDAD } \\
\text { ANCA }\end{array}$ \\
\hline & & & & & \multicolumn{4}{|c|}{ TESI, 15(2), 2014, pp. 152-172 } \\
\hline \multicolumn{9}{|l|}{$\begin{array}{l}\text { a conseguir el trabajo que } \\
\text { quiero }\end{array}$} \\
\hline \multirow{2}{*}{$\begin{array}{l}\text { Me falta información sobr } \\
\text { las características de la } \\
\text { especialidad } \\
\text { Tengo la información } \\
\text { académico-profesional qu } \\
\text { me hace falta }\end{array}$} & 49,8 & 121 & 27,8 & 43 & 23,4 & 164 & 26,5 & $\begin{array}{c}-0,859 \\
\mathrm{p} \leq, 195\end{array}$ \\
\hline & 4,3 & 9 & 2,1 & 5 & 2,7 & 14 & 2,3 & $\begin{array}{c}-0,983 \\
\mathrm{p} \leq, 162\end{array}$ \\
\hline Total Ress & & 436 & 100 & 184 & 100 & 620 & 100 & \\
\hline
\end{tabular}

Al margen de los análisis de cada una de las dimensiones, se llevaron a cabo otros análisis específicos dirigidos a conocer si había relación entre las Dificultades percibidas en el proceso de toma de decisiones y el Grado de decisión sobre la especialidad o salida profesional. Se encontró relación directa (Coeficiente de

Contingencia $\left.=0,389 ; \chi^{2}(1)=57,676 ; \mathrm{p} \leq 0,000\right)$ entre la respuesta 1 al ítem 6 ("tengo muy claro lo que quiero hacer") y la respuesta 5 del ítem 9 ("no tengo dificultades personales de decisión"), tanto entre los estudiantes de segundo como entre los de quinto la relación fue similar. Una interpretación posible de este resultado es que los alumnos que no tienen dificultades en la toma de decisiones personales, tampoco las tienen a nivel vocacional.

\section{4.- CONCLUSIONES}

El CBU ha mostrado ser un instrumento útil para esclarecer los principales indicadores del desarrollo vocacional a la vez que es de sencilla y corta aplicación y fácil de corregir. Las diferentes experiencias, conductas y hechos que en él se recogen nos permiten conocer su eficacia a la hora de planificar la trayectoria evolutiva académica y la preparación profesional de nuestros estudiantes.

En relación con la Motivación o Intereses por la opción universitaria escogida, podemos concluir que los estudiantes de Psicología llegan a la facultad presentando un elevado interés por estos estudios por un lado, son los que habían elegido en primera opción y por otro, deseaban cursarlos desde su época de estudiantes de secundaria. Sin embargo, a pesar de este interés vocacional inicial, las tensiones, incomodidades y expectativas de futuro que aparecen a medida que se aproxima el final de los estudios, se traducen en una ligera disminución de su satisfacción con los mismos. La diferencia encontrada en términos de satisfacción entre los alumnos de segundo y de quinto, podría deberse a la inseguridad del estudiante sobre la manera de enfrentarse al inminente mundo profesional, así como a la falta de consecución de las expectativas que tenían sobre los estudios.

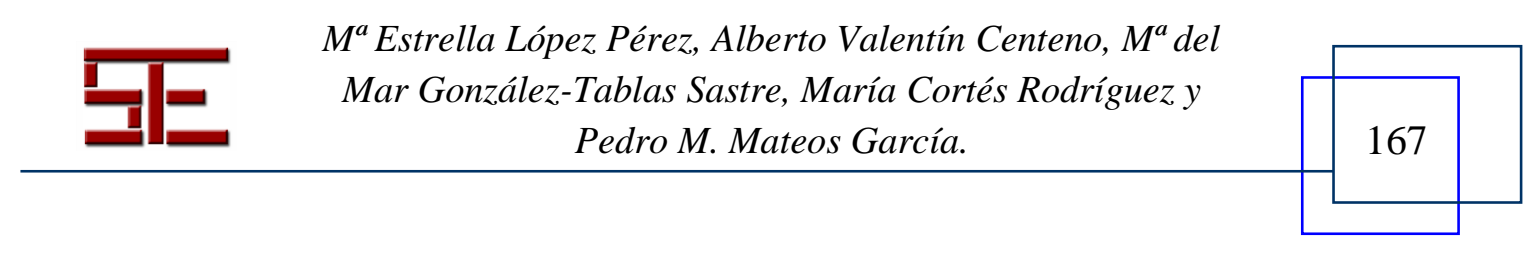




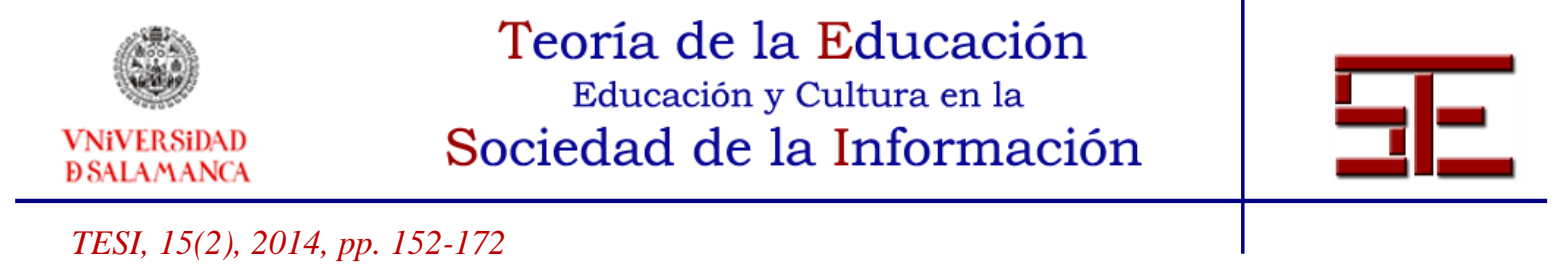

Los datos relacionados con la Toma de Decisiones Académicas o Preprofesionales nos muestran que los estudiantes de Psicología, a lo largo de sus estudios, eligen aquellas asignaturas que le interesan por los contenidos y por la relación con la especialidad a la que quieren dedicarse. No obstante, los alumnos de quinto son más prácticos a la hora de optar al Practicum que los de segundo, al tener en cuenta otros aspectos como son las tareas que se desarrollan, el horario o la proximidad de los centros de prácticas. Cabe apuntar que ante los cambios actuales de nuestra sociedad, tomar decisiones académicas y preprofesionales cada vez es una tarea más compleja para nuestros estudiantes, la incertidumbre creciente sobre su futuro profesional es un hecho que posiblemente marque una clara diferencia en el planteamiento a la hora de enfrentarse a cuestiones del tipo ¿Qué voy a hacer? o ¿A qué me quiero dedicar?

Al analizar el Grado de Decisión sobre la Especialidad o Salida Profesional encontramos que muchos alumnos tienen clara la especialidad o especialidades que les gustan dentro de la psicología, concretamente la Psicología Clínica y de la Salud, seguido de la rama de Psicología Social. Además, en relación con la especialidad, tienen claro que cuando terminen sus estudios necesitan hacer un Máster o Posgrado para dedicarse a ella. Pero, de nuevo, encontramos diferencias entre los alumnos de último curso y los de segundo, son más los alumnos de quinto que no tienen decidido nada y que afirman que necesitan ayuda para poder tomar esta decisión. Estos datos indican que al inicio de los estudios la forma de ver las dimensiones laborales y de especialización de la psicología es más clara y segura que la que presentan los alumnos que están finalizando. Puede deberse a que a medida que van completando su formación van descubriendo más las deficiencias y desconocimientos que tienen. En este momento los estudiantes se plantean dudas sobre su capacidad para enfrentarse al mundo laboral y la necesidad de seguir formándose, de ahí que la mayoría consideren necesario una formación especializada una vez que finalizan sus estudios de psicología.

Por su parte, los datos que recogen información sobre la Experiencia Laboral de nuestros estudiantes apuntan a que en algún momento han tenido un trabajo eventual durante sus estudios, pero son muy pocos los estudiantes que trabajan en algo relacionado con la Psicología. La información recogida por el cuestionario sobre la actividad profesional desempeñada presenta notables limitaciones pues podría perfeccionarse concretando las características de tal actividad (tipo de contrato, dedicación, ámbito laboral, etc.), lo cual permitiría la posible extracción de conclusiones más sólidas sobre la importancia que tiene este aspecto en la orientación vocacional.

El último indicador muestra las Dificultades Percibidas en el proceso de Toma de Decisiones, encontrando que un número importante de alumnos afirman que les falta información sobre la especialidad a la que se quieren dedicar y desconocen cuáles son las opciones que más les conviene para decidirse por una especialidad. De nuevo, los estudiantes de último curso presentan más dificultades, manifestando que ninguna

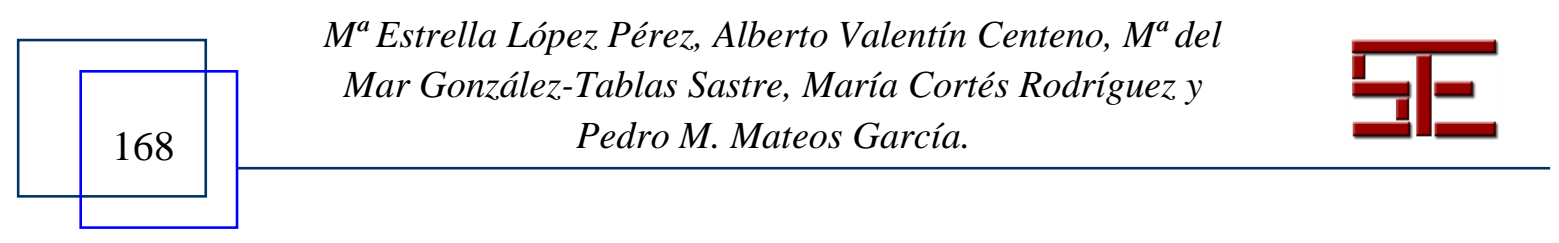




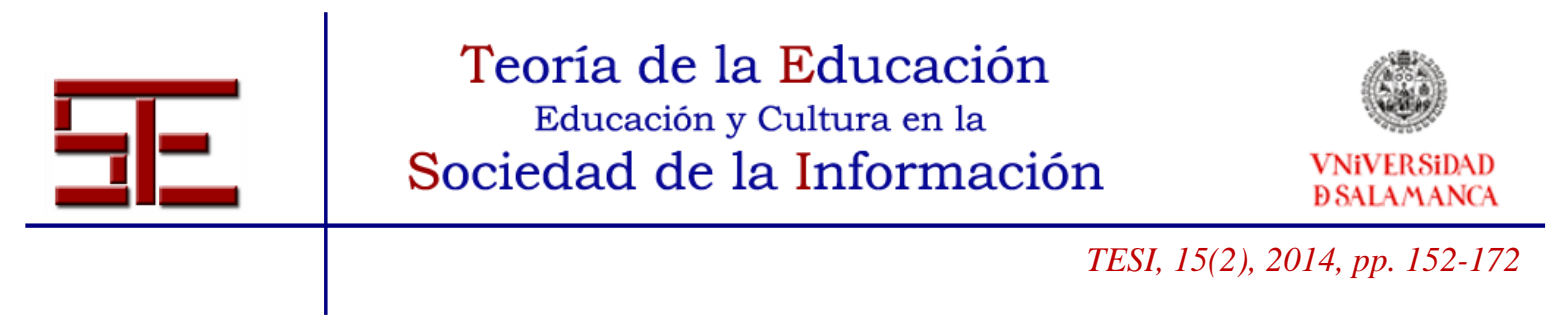

opción les satisface plenamente o que no tienen claro la opción que más les podría interesar. Estos aspectos detectan los problemas que los estudiantes experimentan por la posible falta de orientación necesaria para ayudarles a tomar una decisión adecuada, labor que deberían realizar los servicios de orientación al ser una de sus funciones principales.

La comparación realizada con los resultados del trabajo de Rocabert et al. (2007), dejando al margen las limitaciones que imponen sus diferencias, ha puesto de manifiesto dos muestras de estudiantes universitarios que difieren en algunos aspectos relacionados con la toma de decisiones vocacional. Nuestros alumnos han elegido esta titulación a edades más tempranas que la muestra más heterogénea estudiada por los autores, aspecto que puede estar relacionado con el carácter vocacional de los estudios de Psicología. Además, la preocupación por tomar decisiones relacionadas con la especialidad es mayor también en estos estudiantes, frente a la muestra del trabajo de Rocabert y sus colaboradores. Y, por último, destacamos que sus alumnos, de varias titulaciones, tienen decidido ejercer la profesión y cuentan con la información académico-profesional que necesitan para tomar decisiones, frente a nuestros estudiantes, quienes piensan que necesitan más formación después de la carrera y, además, no cuentan con la información que necesitan para tomar las decisiones necesarias preprofesionales.

El interés inicial por los estudios de Psicología y la ilusión por poder cursar la titulación que habían elegido hace que los estudiantes de los primeros cursos se enfrenten a los estudios de forma más optimista de cara al camino que tienen que elegir para poder dedicarse a la especialidad que quieren. Además, la toma de decisiones en estos primeros cursos son mínimas y el estudiante considera que la facultad va a facilitarle información y formación sobre los recursos que debe adquirir. Empero, nos encontramos con alumnos de último curso que tienen que tomar decisiones que van a afectar de manera decisiva a su futuro profesional, insatisfechos con los estudios y que todavía no tienen decidido que van a hacer cuando terminen. En este momento perciben más dificultades, necesitan información y se dan cuenta de que la orientación recibida no ha sido suficiente para afrontar este futuro con seguridad. Esta falta de eficacia a la hora de prepararse para la especialización o para el mundo laboral es una de las razones que nos llevan a proponer posibles mejoras de los servicios de orientación actuales y la necesidad de llevar a cabo una labor tutorial encaminada al desarrollo académico, profesional y personal desde el inicio de los estudios.

En definitiva, estos resultados indican la necesidad de ofrecer en cada titulación una orientación personalizada sobre la especialidad y formación de postgrado que cada alumno debería recibir en función de sus deseos y capacidades. Esta labor podría llevarse a cabo a través de la acción tutorial, como apunta la adaptación al EEES, encaminada al desarrollo académico, profesional y personal desde el inicio de los

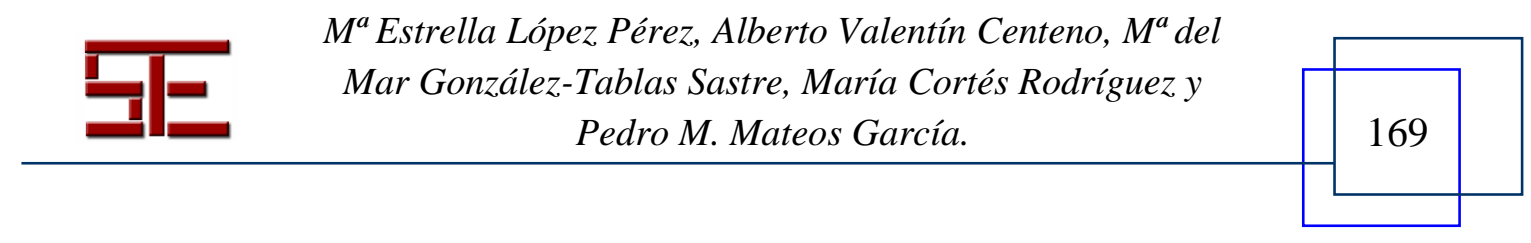




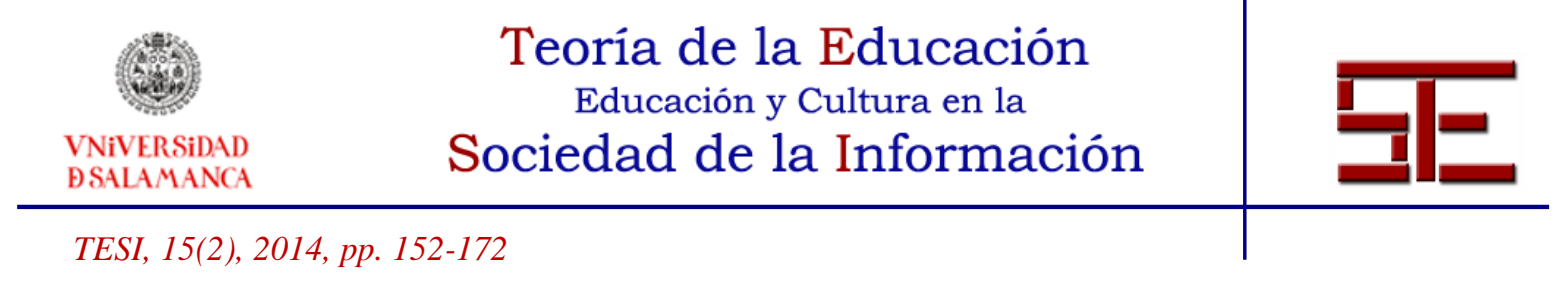

estudios, pero además, el análisis de las actuales Unidades de Orientación nos ayudaría a mejorar sus servicios, garantizando el derecho del estudiante a ser orientado durante su paso por la educación superior, a la vez que serían un poderoso recurso para salvar las dificultades y esclarecer las dudas sobre su futuro en este periodo tan difícil de su vida como es la transición al mundo laboral.

\section{5.- REFERENCIAS BIBLIOGRÁFICAS}

Álvarez, V. (2001). La orientación en centros Universitarios como indicador de calidad. Revista Electrónica Ágora Digital, 2, 1-13. Recuperado de http://www.uhu.es/agora/version01/digital/numeros/02/02-

articulos/monografico/alvarez_rojo.PDF.

Bausela, E. B. (2012). Méndez, L. (2011). Prácticum de Psicología. La formación de profesionales en la enseñanza no presencial. Madrid: Sanz y Torres, pp. 210. Revista Latinoamericana de Psicología, 44 (3), 187-190.

Bricall, J. (Coord.) (2000). Informe Universidad 2000. Madrid: Patronato de la Conferencia de Rectores.

Cárcamo, L., Contreras, P., Muñoz, O. \& Nesbert, F. (2008). Comparaciones en las percepciones de futuro de los Universitarios chilenos y catalanes. Revista Electrónica de Psicología Iztacala 11 (1), marzo, 116-140. Recuperado de http://www.iztacala.unam.mx/carreras/psicologia/psiclin/vol11num1/vol11No1Art7.pdf

Cid, A., Pérez, A. \& Sarmiento, J. A. (2011). La Tutoría en el Practicum. Revisión de la literatura. Revista de Educación. 354, enero-Abril, 127-154.

Gabinete de Estudios Sociológicos y Estadística. Vicerrectorado de Ordenación Académica y Planificación Estratégica. Perfil de los alumnos de nuevo ingreso curso 2005-2006. Característica y opiniones de los alumnos que Ingresaron en la Universidad Politécnica de Madrid (UPM). Abril 2006. Recuperado de http://www.upm.es/sfs/Rectorado/Vicerrectorado\%20de\%20Ordenacion\%20Academica $\% 20 \mathrm{y} \% 20 \mathrm{Planificacion} \% 20$ Estrategica/Compromiso\%20con\%201a\%20Calidad/Enlaces \%20y\%20acceso\%20a\%20documentos/Informe_Demanda_2005-06.pdf.

Jiménez, A. (2007). Un Modelo creativo para la toma de decisiones en orientación profesional universitaria. Revista Galego-Portuguesa de Psicología e Educación, 17 $(1,2)$, Año 13, 1138-1663.

López, E., Valentín, A. \& González-Tablas, M. M. (2012). De las condiciones del Practicum de la licenciatura en psicología a las competencias del grado: análisis de las valoraciones de los alumnos. En Aportaciones recientes al estudio de la Motivación y

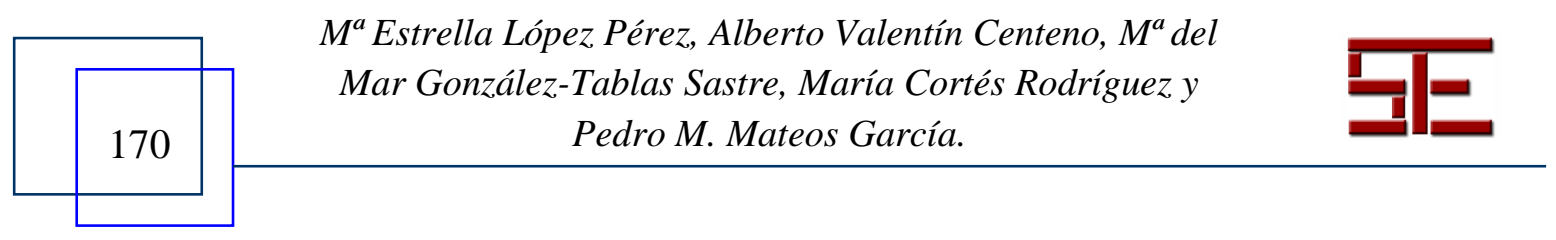




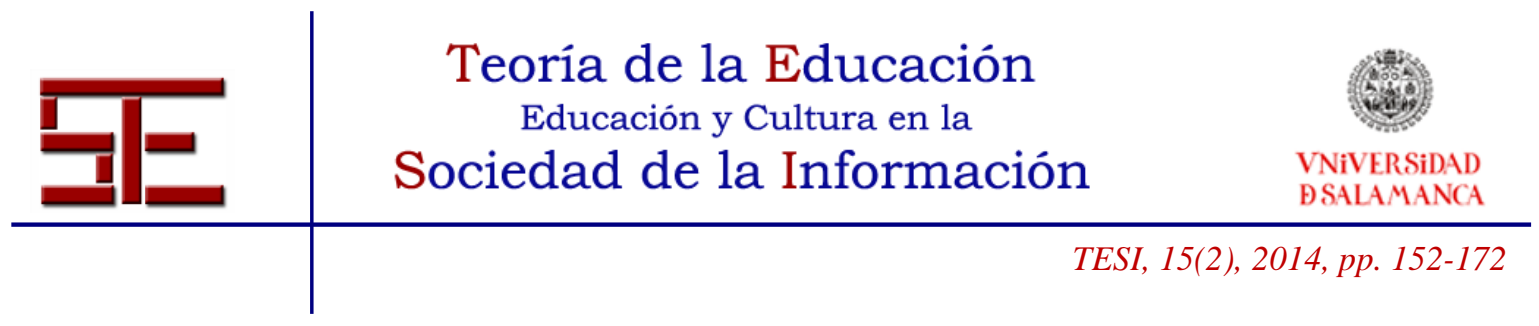

las emociones (pp. 41-48). Sevilla: Fénix Editora. Recuperado de http://web.usal.es/ meilan/Art_2012_MP\%20e\%20inhibicion_CADIZ.pdf.

Martínez, E. \& Raposo, M. (2011). Funciones generales de la tutoría en el Practicum: Entre la realidad y el deseo en el desempeño de la acción tutorial. Revista de Educación. 354, enero-abril, 155-181.

Pérez, M. (2010). ¿Puede un docente sustituir en su rol profesional al Psicólogo Educativo? Papeles del Psicólogo, 31 (2), 150-161.

Rivas, F. (2003). Grupos y patrones vocacionales. En F. Rivas (Ed.), Asesoramiento vocacional. Teoría, práctica e instrumentación (pp. 313-351). Barcelona: Ariel.

Rivas, F., Martín, E. \& De Asís Martín, F. (2008). Conducta Vocacional de estudiantes universitarios españoles. Revista de Orientación Educativa, 22 (40), 13-29.

Rivas, F., Tormos, M.J., \& Martínez, B. (2005). Tecnología informática en asesoramiento vocacional. Psicothema, 17 (3), 382-389.

Rocabert, E. (2005). Biodatos. En F. Rivas \& E. Rocabert. (2005). Conducta vocacional y asesoramiento profesional de los estudiantes universitarios: Protocolo informático para la autoayuda preprofesional. Informe final del proyecto de investigación $\mathrm{I}+\mathrm{D}$ BSO2001-3150, financiado por el Ministerio de Ciencia y Tecnología.

Rocabert, E., Descals, A. \& Gómez, A. (2007). Los Biodatos como indicadores de la conducta vocacional universitaria: Elaboración de un instrumento de evaluación. Electronic Journal of Research in Educational Psychology, 5 (1), 15-36. Recuperado de http://www.investigacion-psicopedagogica.org/revista/new/ContadorArticulo.php?156.

Romero, C., Zurita, F. \& Zurita, M. F. (2010). Autonomía y orientación en el Espacio Europeo de Educación Superior mediante el portafolio y la tutoría. ESE. Estudios sobre Educación, 19, 261-282. Recuperado de http://hdl.handle.net/10171/18322.

Salanova, M., Martínez, I., Bresó, E., Llorens, S. \& Grau, R. (2005). Bienestar psicológico en estudiantes universitarios: facilitadores y obstaculizadores del desempeño académico. Anales de Psicología, 21 (1), junio, 170-180.

Salmerón, H. (2001). Los Servicios de Orientación en la Universidad. Procesos de creación y desarrollo. Revista Electrónica Ágora Digital, 2, 1-18. Recuperado de http://www.uhu.es/agora/version01/digital/numeros/02/02articulos/monografico/salmeron.PDF.

Siegel, S. (1970). Estadística no paramétrica aplicada a las ciencias de la conducta. México: Trillas.

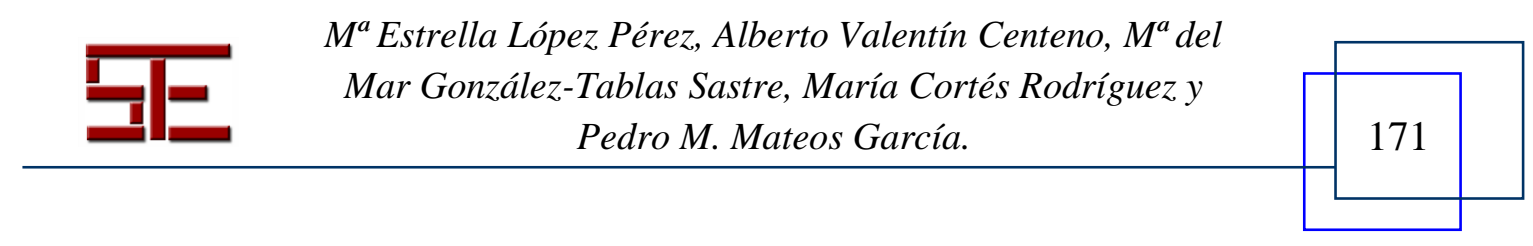




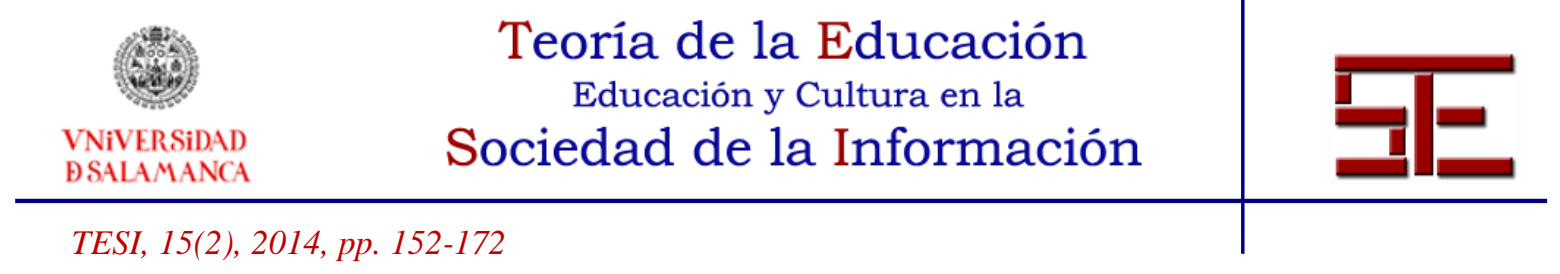

Valentín, A., Mateos, P. M., González-Tablas, M. M., Pérez, L., López, E. \& García, I. (2013). Motivation and learning strategies in the use of ICTs among university students. Computers \& Education, 61, 52-58. doi:10.1016/j.compedu.2012.09.008.

Vázquez, A. \& Manassero, M. (2009). Expectativas sobre un trabajo futuro y vocaciones científicas en estudiantes de educación secundaria. Revista Electrónica de Investigación Educativa, 11 (1). Recuperado de http://redie.uabc.mx/vol11no1/contenido-vazquez4.html.

Vidal, M. \& Fernández, B (2009). Orientación Vocacional. Educación Médica Superior, 23 (2), 1-11.

Vidal Ledo, M., \& Fernández Oliva, B. (2009). Orientación vocacional. Educación Médica Superior, 23(2), 0-0. Recuperado de http://scielo.sld.cu/pdf/ems/v23n2/ems11209.pdf.

Villena, M. D., Muñoz-García, A., Polo, T. \& Jiménez, J. (2010). Organización de la Orientación Europea de Educación Superior: La Unidad de Orientación de Centro. Revista de Investigación Educativa, 8 (3), 329-340.

Para citar el presente artículo puede utilizar la siguiente referencia:

López Pérez, M. E., Valentín Centeno, A., González-Tablas Sastre, M. M., Cortés Rodríguez, M. y Mateos García, P. M. (2014). Análisis de la conducta vocacional en estudiantes de psicología. Revista Teoría de la Educación: Educación y Cultura en la Sociedad de la Información. 15(2), 152-172 [Fecha de consulta: dd/mm/aaaa].

http://campus.usal.es/ revistas_trabajo/index.php/revistatesi/article/view/11891

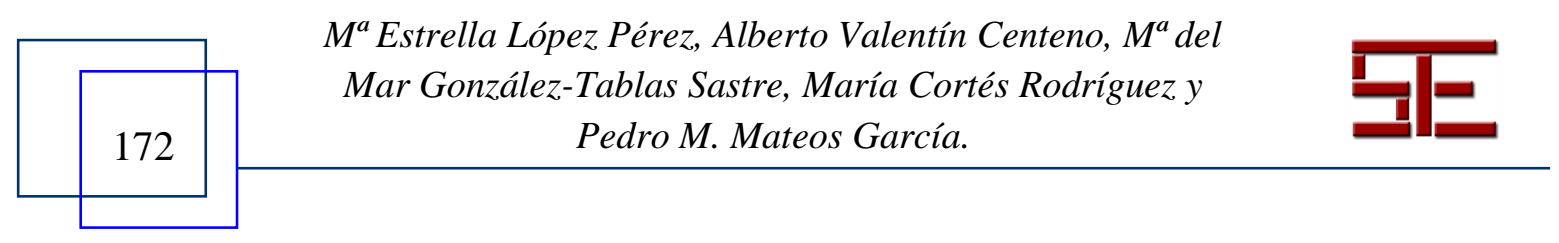

